\title{
Sunspot splitting triggering an eruptive flare
}

\author{
Rohan E. Louis ${ }^{1}$, Klaus G. Puschmann ${ }^{1}$, Bernhard Kliem²,3,4 Horst Balthasar $^{1}$, and Carsten Denker ${ }^{1}$ \\ 1 Leibniz-Institut für Astrophysik Potsdam (AIP), an der Sternwarte 16, 14482 Potsdam, Germany \\ e-mail: rlouis@aip.de \\ 2 Yunnan Astronomical Observatory, Chinese Academy of Sciences, 650011 Kunming, PR China \\ 3 Institute of Physics and Astronomy, University of Potsdam, 14476 Potsdam, Germany \\ ${ }^{4}$ Mullard Space Science Laboratory, University College London, Holmbury St. Mary, Dorking RH5 6NT, UK
}

Received 15 January 2013 / Accepted 16 November 2013

\begin{abstract}
Aims. We investigate how the splitting of the leading sunspot and associated flux emergence and cancellation in active region NOAA 11515 caused an eruptive M5.6 flare on 2012 July 2.

Methods. Continuum intensity, line-of-sight magnetogram, and dopplergram data of the Helioseismic and Magnetic Imager were employed to analyse the photospheric evolution. Filtergrams in $\mathrm{H} \alpha$ and He I $10830 \AA$ of the Chromospheric Telescope at the Observatorio del Teide, Tenerife, track the evolution of the flare. The corresponding coronal conditions were derived from $171 \AA$ and $304 \AA$ images of the Atmospheric Imaging Assembly. Local correlation tracking was utilized to determine shear flows.

Results. Emerging flux formed a neutral line ahead of the leading sunspot and new satellite spots. The sunspot splitting caused a longlasting flow towards this neutral line, where a filament formed. Further flux emergence, partly of mixed polarity, as well as episodes of flux cancellation occurred repeatedly at the neutral line. Following a nearby C-class precursor flare with signs of interaction with the filament, the filament erupted nearly simultaneously with the onset of the M5.6 flare and evolved into a coronal mass ejection. The sunspot stretched without forming a light bridge, splitting unusually fast (within about a day, complete $\approx 6 \mathrm{~h}$ after the eruption) in two nearly equal parts. The front part separated strongly from the active region to approach the neighbouring active region where all its coronal magnetic connections were rooted. It also rotated rapidly (by $4.9^{\circ} \mathrm{h}^{-1}$ ) and caused significant shear flows at its edge.

Conclusions. The eruption resulted from a complex sequence of processes in the (sub-)photosphere and corona. The persistent flows towards the neutral line likely caused the formation of a flux rope that held the filament. These flows, their associated flux cancellation, the emerging flux, and the precursor flare all contributed to the destabilization of the flux rope. We interpret the sunspot splitting as the separation of two flux bundles differently rooted in the convection zone and only temporarily joined in the spot. This explains the rotation as the continued rise of the separating flux, and it implies that at least this part of the sunspot was still connected to its roots deep in the convection zone.
\end{abstract}

Key words. Sun: flares - sunspots - Sun: photosphere - Sun: chromosphere - techniques: photometric

\section{Introduction}

Solar flares are energetic phenomena involving plasma heating, particle acceleration, and the release of electromagnetic energy spanning the range from X-rays to radio wavelengths. Eruptive flares are part of a coronal disruption that leads to a coronal mass ejection (CME) and also often includes the eruption of a filament or prominence. Such disruptions, also referred to as solar eruptions, originate in regions of a highly non-potential coronal field. They have a sudden onset and develop on much shorter time scales than the evolutionary time scales of the photosphere on the spatial scales involved. Thus, they include the gradual storage of free magnetic energy in the coronal field by changes in the photospheric boundary and the sudden loss of equilibrium, or onset of instability, at a critical point in the evolution of the coronal field (Forbes 2010). The resulting release of free magnetic energy returns the field to a more potential, less stressed configuration.

Eruptions occur invariably in highly sheared coronal field above photospheric polarity inversion (neutral) lines, especially if there is also a strong gradient of the magnetic field (Martres et al. 1968; Hagyard et al. 1990; Wang 2006). Such regions

\footnotetext{
* Movie available in electronic form at http://www . aanda.org
}

show a filament channel in the chromosphere and often contain a filament or prominence in the corona above (Martin 1998). Although the complete magnetic structure of filaments remains a mystery, the widely adopted assumption of a weakly twisted magnetic flux rope holding the cool material is consistent with the majority of filament properties, especially with the observed, typically inverse field direction in their bottom part (Mackay et al. 2010). The formation and instability of a flux rope is a key element of storage-and-release eruption models (Forbes \& Priest 1995; Amari et al. 2003; Mackay \& van Ballegooijen 2006; Kliem \& Török 2006). The resulting rise of the flux rope forms a vertical current sheet underneath, where flare reconnection is triggered (Lin \& Forbes 2000).

The changes in the magnetic field that precede and lead to such eruptions are complex and can vary greatly from event to event. They may involve the emergence, shearing and/or twisting, and cancellation of flux in the photosphere, as well as smallscale precursor activities in the corona. Various combinations of these processes have been observed, and despite intense study, a clear picture of their roles in triggering eruptions has not yet emerged (e.g., Zhang et al. 2008b). By performing a detailed analysis of a comprehensively observed, relatively strong eruptive flare, we address the relationship between the evolution of 
the photospheric flux distribution and the corresponding evolution of the coronal field leading to the eruption. This event features the splitting of the source region's leading sunspot as a special driving factor and is additionally characterized by considerable complexity in the photospheric changes. However, we find that the evolution towards the eruptive flare can be understood in terms of the three basic photospheric driving processes mentioned above acting in combination. Still, the sunspot splitting was unusual, intriguing on its own. We briefly characterize the basic photospheric driving processes in the following.

Flares often occur in emerging flux regions, where newly emerged fields appear in a region of pre-existing flux (e.g., Rust 1972; Martin et al. 1982; Demoulin et al. 1993; Feynman \& Martin 1995; Li et al. 2000; Sakajiri et al. 2004; Schrijver 2009; Sun et al. 2012). Many authors suggest that the emergence leads to the formation of a magnetic flux rope in the corona, either by bodily emergence (Low 1996; Lites 2005) or by reconnection within an emerged magnetic arcade (Manchester et al. 2004; Archontis \& Hood 2010). See Vargas Domínguez et al. (2012) for a discussion of these competing concepts. It has been suggested that shear flows at the neutral line are intimately connected with the emergence process, as the primary driver of the flux rope formation (Manchester et al. 2004). However, it is not clear whether the connection is as universal as has been suggested.

Horizontal shear flows in the photosphere are often seen in any evolutionary phase of an active region, as well as between adjacent active regions. They can be derived from photospheric data (Deng et al. 2006; Tan et al. 2009; Liu \& Schuck 2012) or be inferred from the changing appearance of coronal loop arcades (Green et al. 2011). A direct relationship with flaring activity has been established in these and in may other cases. It is not yet clear whether flares and CMEs are triggered in highly sheared arcade fields (Karpen et al. 2012), although no large-scale instability of this configuration is known or whether the high shear first leads to the formation of a magnetic flux rope, whose instability then causes the eruption.

Flares can also be triggered by the rapid rotation of sunspots (Zhang et al. 2007; Yan et al. 2009; Min \& Chae 2009; Jiang et al. 2012; Yan et al. 2012; Török et al. 2013), which twists the coronal field, such that the helical kink instability may be triggered in cases of very strong rotation. Rotational motions rarely occur in isolated sunspots, so in most cases they also shear the field rooted near the periphery of the rotating spot (e.g., Schrijver et al. 2008).

Finally, flux cancellation is an important mechanism for triggering solar flares (Livi et al. 1989; Sterling et al. 2010; Green et al. 2011; Savcheva et al. 2012; Burtseva \& Petrie 2013). In particular, Zhang et al. (2001) located the initial flare brightenings and filament disturbances of a major eruptive event exactly at the sites of flux cancellation. The link between these phenomena is usually considered to be the formation of a flux rope, which is expected if the cancelling photospheric flux patches lie at the base of sheared coronal flux (van Ballegooijen \& Martens 1989; Mackay \& van Ballegooijen 2006; Aulanier et al. 2010; Amari et al. 2011). The cancellation then involves reconnection of the sheared field low in the atmosphere, as observationally verified by Wang \& Shi (1993). This produces a flux rope in the corona. Flux cancellation at the neutral line is a natural result of the flux dispersal in the decay phase of active regions, so this process is considered to be the typical driver of eruptions in decaying active regions.

In this paper we analyse an eruptive M5.6 flare originating in a complex active region. We employ multi-wavelength observations covering the wide range of scales involved in the photosphere, chromosphere, upper transition region, and corona. The causal relationships between the photospheric and coronal phenomena lie in the main focus of this investigation, but we also address the possible origin of the sunspot splitting. The paper is organized as follows. The observations and procedures for data reduction are described in Sect. 2. We describe the results of our analysis in Sect. 3. The discussion and conclusion are presented in Sects. 4 and 5, respectively.

\section{Observations and data processing}

For this investigation we employed full-disc solar observations from the Solar Dynamics Observatory (SDO; Pesnell et al. 2012) which include data from the Helioseismic and Magnetic Imager (HMI, Schou et al. 2012) and the Atmospheric Imaging Assembly (AIA, Lemen et al. 2012) for NOAA active region (AR) 11515 during 2012 July 1-4. The HMI data set consists of $4 \mathrm{k} \times 4 \mathrm{k}$-pixel images of continuum intensity, dopplergrams, and line-of-sight (LOS) magnetograms derived from the photospheric Fe I $617.3 \mathrm{~nm}$ line at a cadence of $12 \mathrm{~min}$ and a spatial sampling of $0.5^{\prime \prime} \mathrm{pixel}^{-1}$. The continuum images were corrected for limb darkening using a fifth-degree polynomial fit to the average radial profile. Projection effects were compensated for using the method described by Gary \& Hagyard (1990). The dopplergrams and magnetograms were corrected for the $\cos \Theta$ factor (where $\Theta$ is the heliocentric angle) and for projection effects similar to the continuum images. All three sets of images were coaligned using a two-dimensional cross correlation routine. We also used 4k × 4k-pixel, AIA Fe IX $171 \AA$ and He II $304 \AA$ images, which have a spatial sampling of $0.6^{\prime \prime}$ pixel $^{-1}$ and a cadence of up to $12 \mathrm{~s}$. These wavelengths correspond to temperatures of $\log (T)=5.8$ and $\log (T)=4.7$.

We investigated the chromosphere of the active region using observations from the Chromospheric Telescope (ChroTel, Kentischer et al. 2008; Bethge et al. 2011, 2012a) operated next to the German Vacuum Tower Telescope (VTT; Schröter et al. 1985) at the Observatorio del Teide, Tenerife, Spain. ChroTel provides narrow-band filtergrams in three wavelengths, namely, Ca II K $3933.7 \AA, H \alpha 6562.8 \AA$, and He I $10830.3 \AA$, using three separate Lyot filters. The passbands of the Lyot filters at the above wavelengths are $0.3,0.5$, and $1.3 \AA$, respectively. Around $10830 \AA$, a tunable filter was employed, which delivers filtergrams at seven fixed wavelengths (numbered 1-7) with non-equidistant wavelength spacings. This arrangement facilitates the retrieval of LOS velocities. The $2 \mathrm{k} \times 2 \mathrm{k}$-pixel images have a spatial sampling of about $1^{\prime \prime}$ pixel $^{-1}$ and exposure times of 1000, 300, and $500 \mathrm{~ms}$, respectively. On July 2, ChroTel provided filtergrams in all three wavelengths with a cadence of 3 min from 6:54 UT to 17:06 UT. The ChroTel images were corrected for limb darkening and rescaled to match the HMI images. The chromospheric filtergrams were deprojected and co-aligned using the same scheme as mentioned above.

\section{Results}

\subsection{General evolution of active region NOAA 11515}

The leading sunspot of AR 11515 became clearly visible at the east limb on 2012 June 27 . We analyse its evolution in the period July 1-4 displayed in Figs. 1 and 2 (each with co-temporal panels P1-P24). At the beginning of July 1, the region is located at S19 E28 and has a $\beta \gamma$ magnetic configuration (following the Hale classification, Hale \& Nicholson 1938), consisting 

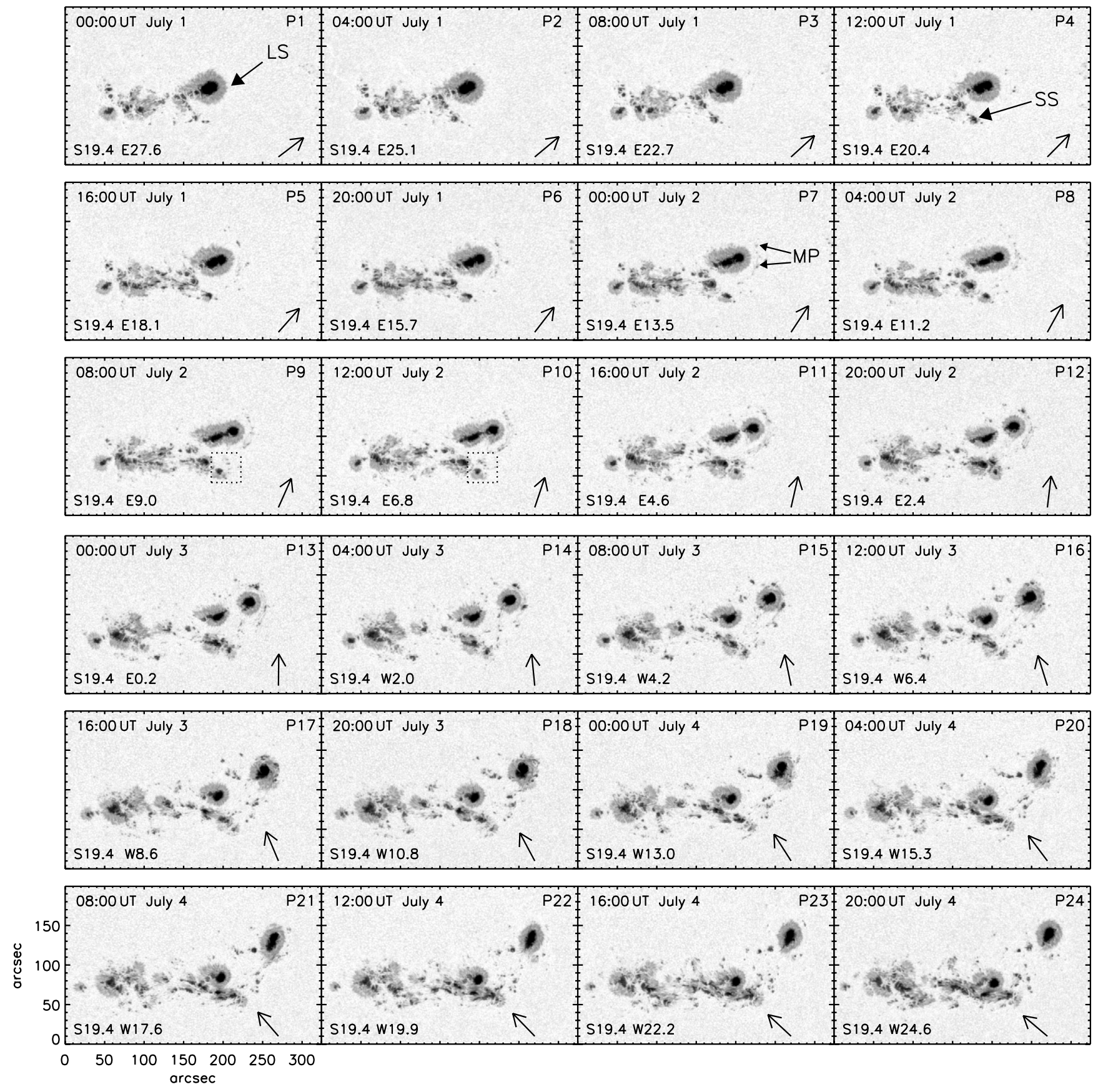

Fig. 1. Time sequence of continuum images from SDO/HMI. The time and date of the observations are indicated in the top left corner, while the position of the active region on the solar disc is shown in the bottom left corner of each panel. The arrow in the lower right corner of each panel points to disc centre. Solar east and north are to the left and top, respectively. Leading sunspot - LS, the satellite group of sunspots - SS, and a region of mixed polarity - MP. The panel number is indicated on the top right corner.

of a leading sunspot of positive polarity followed by a complex group of smaller spots, mostly of negative polarity. From about this time, a group of satellite sunspots with the same polarity as the leading sunspot appears south of the latter (panels P1-P4). Additionally, by $\approx 14: 00 \mathrm{UT}$, the emergence of significant new flux in front of the leading sunspot is obvious (P3-P5). It consists of mixed but dominantly negative polarity, thus forming a new neutral line. This region of flux emergence and its associated neutral line develop into an arc that extends southwards to the emerged satellite polarity (P6-P7). A filament begins to form in the corona along the newly established neutral line around 18:30 UT, as seen in AIA $304 \AA$ images.
In the early part of July 2, the leading sunspot, as well as the small group of satellite sunspots, clearly separated from their more complex trailing spots. During this period, the leading sunspot is seen to stretch, with penumbral filaments on either side moving towards each other near the middle of the spot (P8-P9). The leading edge of the spot thus approaches the newly formed neutral line, a situation previously found to trigger flare activity on a wide range of scales, from a large $\delta$ spot region producing X-class flares (Wang et al. 1991) down to sub-arcsecond scales (Denker \& Wang 1998). Additionally, the arc-shaped region of dominantly negative flux emergence approaches the satellite polarities in a period of more than a 

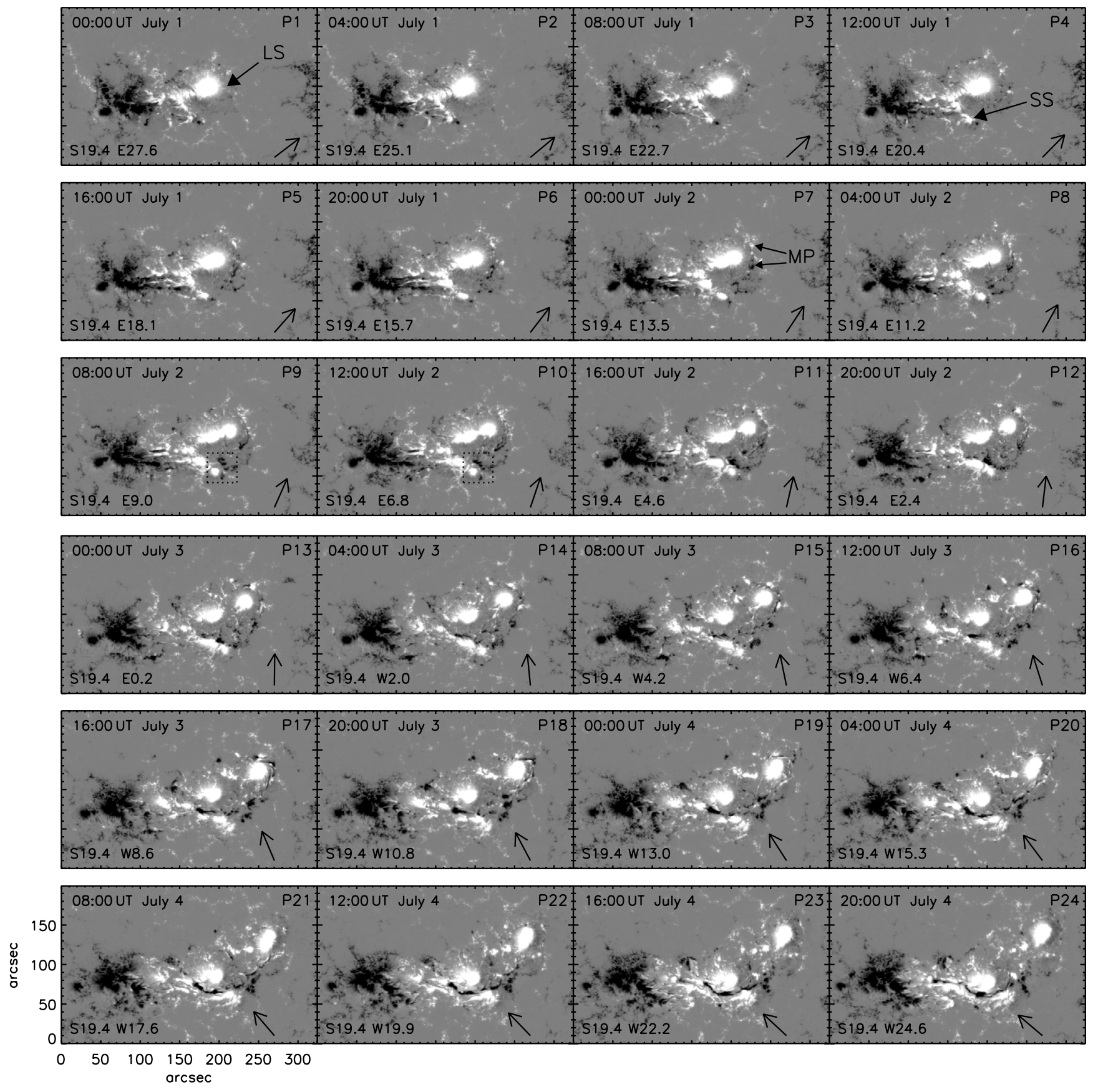

Fig. 2. Same as Fig. 1 but for line-of-sight magnetograms.

day (P5-P15), producing a closely packed configuration that is suggestive of flux cancellation.

At about 10:43 UT on July 2 the filament erupts and simultaneously an M5.6 flare commences. The eruption begins near the satellite polarities (dotted square in P9-P10) but quickly extends along the whole length of the neutral line, and results in a CME.

The stretching of the leading sunspot continues until it splits into two nearly equal parts (P11), and the leading half and its moat separate from the rest of the active region. This "runaway" sunspot exhibits significant proper motion and rotation after its separation, thus continuing to approach the arc-shaped neutral line ahead of it. Episodes of flux cancellation with minority polarity patches ahead of it then show up more clearly. Strong shear flows are observed near the periphery of the runaway sunspot in this phase and subsequently (P17-P19). These shear flows are no longer seen on July 4, and the sunspot drifts further away. The other half of the leading sunspot that remained within the active region merges with the satellite group of sunspots, where a strong neutral line is established early on July 4. This newly established $\beta \gamma \delta$ configuration produces a long series of further flares, including $20 \mathrm{M}$-class flares, several of which cause a Sun quake (Zharkov \& Martínez Oliveros, priv. comm.).

\subsection{The M5.6 flare on 2012 July 2}

Although many M- and C-class flares originate in AR 11515 during its transit on the solar disc, in this paper we only describe and analyse the M5.6 flare and possible precursors associated with the sunspot splitting. The available multi-wavelength data 
cover the evolution of the flare and of the erupting filament comprehensively, both in the chromosphere and corona.

At the time of the eruption, the active region is located at S19 E8 $\left(\Theta=20.7^{\circ}\right)$. In the $1-8 \AA$ soft X-ray band monitored by the Geostationary Operational Environmental Satellite (GOES, Neupert 2011, and references therein), the M5.6 flare starts near 10:43 UT and peaks at 10:52 UT. The flux decreases to the (relatively high) background level of $\sim 10^{-6} \mathrm{~W} \mathrm{~m}^{-2}$ around 11:10 UT, although the flare is eruptive and associated with the filament eruption and subsequent CME. A preceding C2.9 flare started at 10:33 UT and peaked at 10:37 UT. AIA images show it to be associated with a faint, southwards propagating spray, but otherwise there are no indications of ejective behaviour. This suggests that the C2.9 flare is a confined event.

Figure 3 shows the time sequence of $\mathrm{H} \alpha$ (Col. 1) and He I $10830 \AA$ images from ChroTel, which depict the spatial evolution of the flares. Columns 2 to 4 correspond to filtergrams taken at different central wavelength positions, namely 10828.5 , 10830.3, and $10832.1 \AA$. At these wavelengths, the Lyot filter samples the outermost red wing of a photospheric Si I line, the two strongest helium lines, and a water vapour line on the red side of the He I lines, respectively. Column 5 shows the LOS velocity derived from the five innermost filtergrams (nos. 2-6 in Bethge et al. 2011). The derivation of the LOS velocity has been explained in Bethge et al. (2011), wherein the weight factor for each filtergram has to be determined through calibration with spectroscopic data. Since there were no spectroscopic observations of the He I triplet on July 2, we compute the LOS velocity from the mean weight factors determined by Bethge et al. (2011). For this reason, and since we are dealing with a wide range of velocities from several $\mathrm{km} \mathrm{s}^{-1}$ in the region of the flare to less than $2 \mathrm{~km} \mathrm{~s}^{-1}$ in the quiet Sun, we refrain from making quantitative estimates of the velocities.

Panel 1 of Fig. 3 shows the presence of a large filament (white arrow in ChroTel $\mathrm{H} \alpha$ image) following the arc-shaped neutral line in front of the leading spot and extending to the area of the satellite group of sunspots. The length of the filament is about $50^{\prime \prime}$ and the width is about $20^{\prime \prime}$. At the southern end of the filament, there is a compact brightening close to the satellite group of sunspots. Similar brightenings are observed near the northern end of the filament close to the leading sunspot, in the complex negative-polarity part of the active region and in the trailing part of AR 11514 west of the leading sunspot (right side of panel 1 in the ChroTel $\mathrm{H} \alpha$ image). Six minutes later, the satellite group of sunspots is covered by $\mathrm{H} \alpha$ emission that has expanded from the brightening seen earlier (panel 2). Smaller intensity enhancements are seen in ChroTel filtergrams around $10830 \AA$, which are more pronounced in the outer wing of the Si I line and the He I triplet (Cols. 2-3). These appear like small flare kernels (Harvey 1985) and are the signatures of the $\mathrm{C} 2.9$ flare. The $\mathrm{H} \alpha$ emission stays at an enhanced level in the decay phase of this flare, while the He filtergrams are devoid of any emission at 10:42 and 10:48 UT (panels 3-4). The filament is no longer visible in $\mathrm{H} \alpha$ after the peak of the $\mathrm{C}$ class flare (panels 4-5), possibly because of the heating and ionization of its plasma (Yang et al. 2012). We obtain an $\mathrm{H} \alpha$ light curve of the flares from the ChroTel images by calculating the mean $\mathrm{H} \alpha$ intensity within a smaller field of view enclosing the flare emission and excluding dark features such as sunspots and the filament. The comparison with the GOES X-ray light curve in Fig. 4 demonstrates that the brightening around 10:36 UT in the ChroTel images is the $\mathrm{C} 2.9$ flare, which thus has a close spatial association with the M5.6 flare, that is, it is a true precursor event.

The He I emission of the M5.6 flare in the ChroTel filtergrams commences in the impulsive flare phase (panel 5 of Fig. 3). During the peak of the flare, a strand of the erupting filament is seen in $\mathrm{H} \alpha$, while the He filtergrams capture the acceleration of the body of the filament as it erupts. In particular, the first changes in the location of the filament occur between the $10832.1 \AA$ images in panels 3 and 4. The filament eruption is also seen in the He I LOS velocities, which show the bulk of the filament material exhibiting high blue shifts (panels 7-10). One also finds traces of weak red shifts along narrow filament strands, which is consistent with the results of Yang et al. (2012). The flare kernels, which are locations of strong emission, appear red-shifted in the He I LOS velocity maps, possibly due to nonthermal heating of the plasma (panels 5-7). Traces of the filament are observed in $\mathrm{H} \alpha$ after 10:5 UT, indicating subsequent cooling of the filament material (panels 8-10).

The higher cadence of the AIA images allows us to narrow down the onset time of the filament eruption to the interval 10:42-10:43 UT, nearly simultaneous to the onset of the M-class flare. Since the exact onset time of the M-class flare is masked in the soft X-ray light curve by the preceding C-class flare, we cannot determine whether the filament eruption or the M-class flare commenced first. The filament remains visible in the AIA $304 \AA$ images up to about 10:46:30 UT. These show more clearly than the $10832.1 \AA$ images in Fig. 3 that the eruption begins at the southern end of the filament (Fig. 5).

The filament eruption evolves into a southward traveling CME with an estimated ${ }^{1}$ start time near 11:05 UT, angular width of $125^{\circ}$ and projected velocity of $313 \mathrm{~km} \mathrm{~s}^{-1}$. The true velocity must be considerably higher, since the eruption occurs not far from disc centre.

Figure 6 shows the evolution of the C2.9 flare towards the onset of the M5.6 flare as seen in $\mathrm{H} \alpha$. ( The images at this wavelength do not show the whole extent of the filament; see Fig. 5). The C2.9 flare launches an $\mathrm{H} \alpha$ intensity front whose leading edge is tracked by crosses in the top panels of Fig. 6. The intensity front moves at about $20 \mathrm{~km} \mathrm{~s}^{-1}$ towards the filament, traversing its southern part exactly in the interval where it starts to erupt (10:42-10:43 UT) and remaining at this position subsequently. This suggests that an interaction occurred between the precursor flare and the erupting filament.

\subsection{Associated photospheric changes}

In the following subsections, we analyse the photospheric changes in the active region around the time of the M5.6 flare, based on HMI continuum images and LOS magnetograms, and relate them to the activity in the upper atmosphere. A comprehensive picture includes the long-term trends in the region that persist for more than a day, as well as rapid changes shorter than an hour, which occur in close temporal and spatial association with the flare.

The most conspicuous feature in the active region is the splitting of the leading sunspot and the large displacement of its front fragment (Fig. 1). The splitting begins about $20 \mathrm{~h}$ before the M5.6 flare with a pronounced stretching and proceeds across the middle of the spot where the northern and southern penumbral segments approach each other (panels P5-P8 of Fig. 1), but no light bridge appears. The velocity of the front part reaches

Data available at http://cdaw.gsfc.nasa.gov/ 


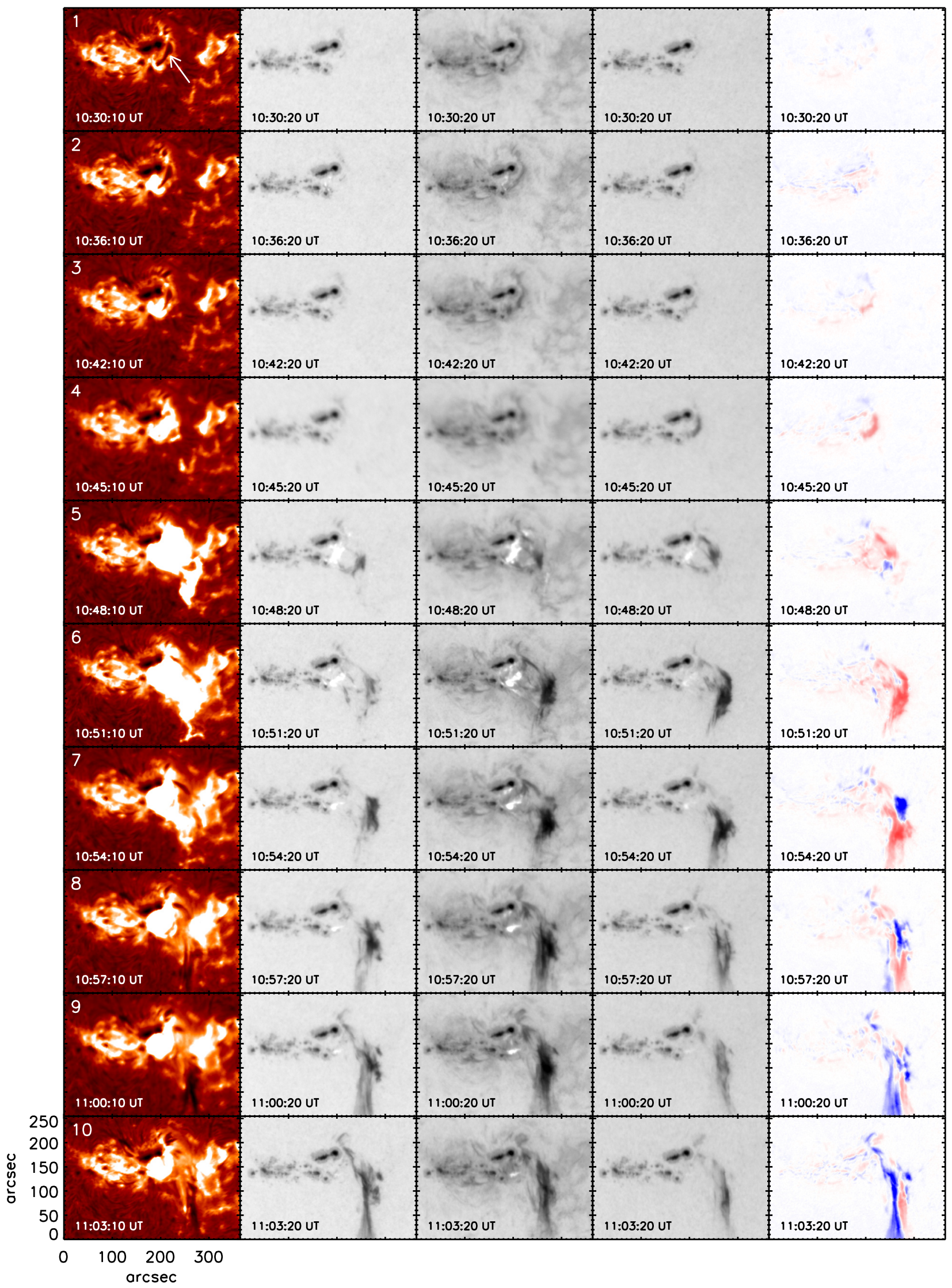

Fig. 3. Temporal evolution of the M5.6 flare on 2012 July 2 as seen in ChroTel observations. The first column shows ChroTel H $\alpha$ filtergrams, and Cols. 2 to 4 correspond to ChroTel filtergrams at $10828.5 \AA$, $10830.3 \AA$, and $10832.1 \AA$, respectively. Column 5 depicts He I LOS velocities derived from the five innermost filtergrams. Blue corresponds to blue shifts and red to red shifts. The panel number is indicated in the top left corner. 


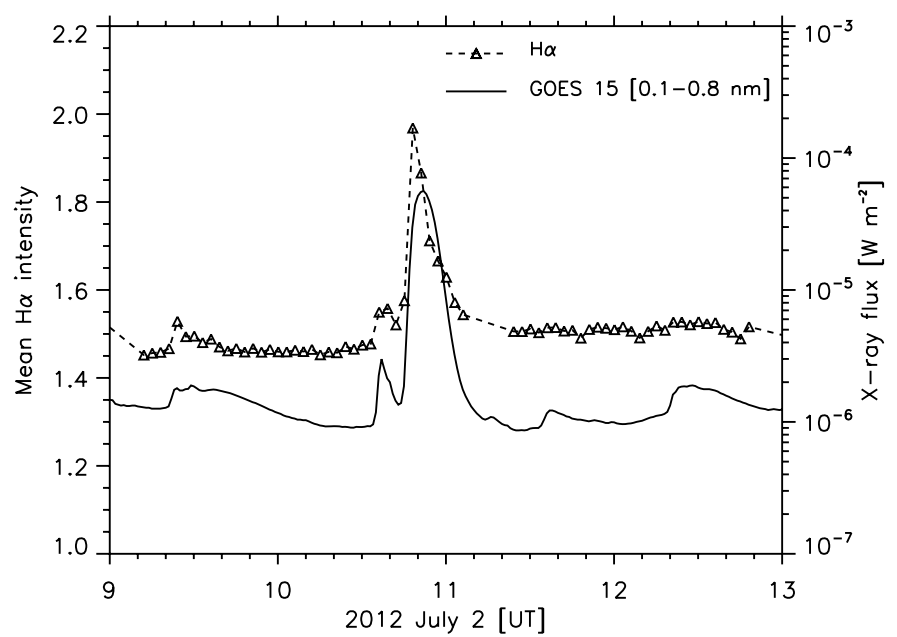

Fig. 4. Temporal evolution of the mean $\mathrm{H} \alpha$ intensity (dashed line with triangle symbols) in the region of the flare as obtained from ChroTel, and the GOES $15 \mathrm{X}$-ray flux in the $0.1-0.8 \mathrm{~nm}$ energy channel (solid line). A small C2.9 flare occurred at 10:37 UT, which is immediately followed by the M5.6 flare at 10:52 UT.

about $300 \mathrm{~m} \mathrm{~s}^{-1}$, and two fragments of nearly equal size are produced about six hours after the flare. The primary component of the front fragment's motion points westwards, i.e. towards the arc-shaped neutral line ahead of it, where one end of the filament is anchored. The separation also has a weaker northward component. Since the neutral line runs roughly in the northwestern direction in this area, the splitting of the spot comprises a shear flow component in addition to the converging flow. This is fully analogous to previously analysed cases of flares caused by spots moving towards newly emerged flux in their vicinity (e.g., Wang et al. 1991; Denker \& Wang 1998). (See the following sections for further development of the split sunspot.)

The evolution towards the eruption also includes many changes in the photospheric flux on smaller scales. Figure 7 illustrates instances of small-scale flux emergence along the neutral line close to the leading sunspot in the box labelled " 1 " in Fig. 6. The yellow circle outlines patches of positive and negative polarity that emerge close to the time of the M5.6 flare. Figure 8 shows the temporal change of the magnetic flux near that neutral line. All flux detected within the yellow contour in box " 1 " in Fig. 6 is plotted, after subtracting the fluxes at 10:00 UT. Most of the yellow contour in Fig. 6 is selected by eye to include all significant contributions and fixed throughout the time series, but the section at the periphery of the splitting spot is determined automatically at the outer penumbra boundary in the continuum image at the time of each measurement. The plot in Fig. 8 shows a close temporal association of flux emergence with the triggering of the $M$ flare. While the peak emergence rates of the two polarities differ moderately $\left(3.7 \times 10^{16} \mathrm{Mx} \mathrm{s}^{-1}\right.$ and $-6.6 \times 10^{16} \mathrm{Mx} \mathrm{s}^{-1}$ from the linear fits in the figure), the total changes preceding the M5.6 flare are about the same, nearly $\pm 3 \times 10^{19} \mathrm{Mx}$.

Complex changes likewise occur near the part of the neutral line north of the satellite spots, i.e. in the area of the precursor flare and the initial filament motion. Both the satellite spots (positive flux) and the adjacent southern end of the arcshaped flux emergence region (negative flux) are active sites of flux emergence prior to and throughout the flares (Sect. 3.1 and Fig. 2). Additionally, these areas of opposite polarity approach each other in a period of about $40 \mathrm{~h}$ that includes the flares (panels P5-P15 in Fig. 2). The latter evolution is
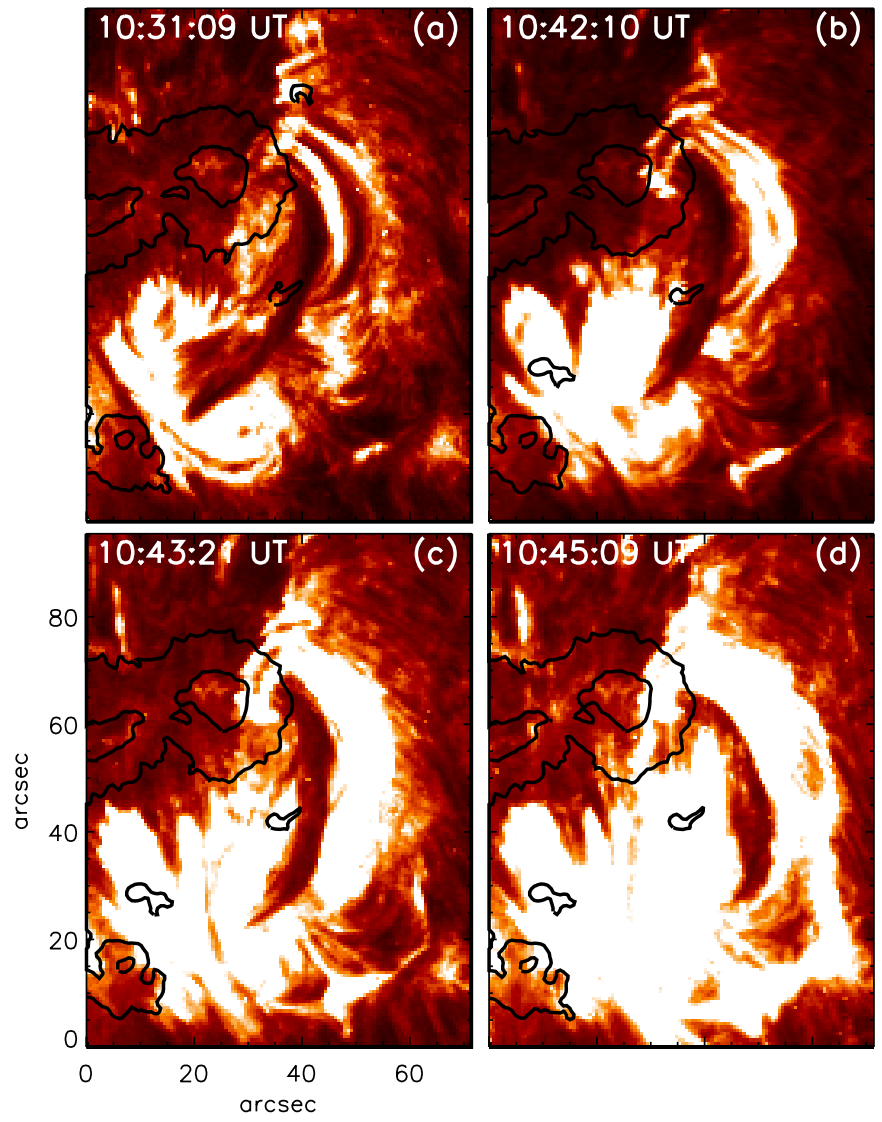

Fig. 5. Sequence of AIA $304 \AA$ images showing the filament immediately before a) and after b)-d) the onset of the eruption.

highly suggestive of flux cancellation, but very difficult to quantify, since the presumed cancellation is superimposed on flux emergence. However, several small-scale flux cancellation episodes embedded in this evolution can be clearly discerned, and we present two of them in the following. The flux cancelled in these episodes is only a small fraction of the whole flux that disappears at this part of the neutral line in the $40 \mathrm{~h}$ interval (compare Fig. 2).

Figure 9 shows a magnified view of a small region marked by the grey dashed square and labelled " 2 " in Fig. 6. Two sites of flux cancellation/disappearance were identified. The two small magnetic patches have been enhanced in flux by a factor of six in this plot for ease of identification. Both patches appear close to 10:00 UT. In the first event, a positive flux patch marked by the violet contour lies adjacent to the newly emerged flux region of opposite polarity, which has been indicated by the white contour. This patch appears to move closer to the latter with a velocity of about $630 \mathrm{~m} \mathrm{~s}^{-1}$, traversing almost the full diameter of the green circle shown in Fig. 9. This velocity is consistent with the range of $300-800 \mathrm{~m} \mathrm{~s}^{-1}$ estimated by Mathew \& Ambastha (2000). The magnetic flux increases slowly from $2.3 \times 10^{18}$ to $3.3 \times 10^{18} \mathrm{Mx}$ in a time span of $30 \mathrm{~min}$ starting at 10:00 UT. However, this is followed by a rapid decrease in flux from 10:30 UT as is evident in Fig. 9, with the patch completely disappearing at 10:39 UT.

The other event identified in the sequence is observed close to the edge of the satellite sunspot's penumbra. There, a negative flux patch, shown by the turquoise contour, appears to be an island surrounded mostly by positive flux. Different from the positive flux patch described above, this negative polarity patch appears to be stationary throughout its lifetime. The initial flux 


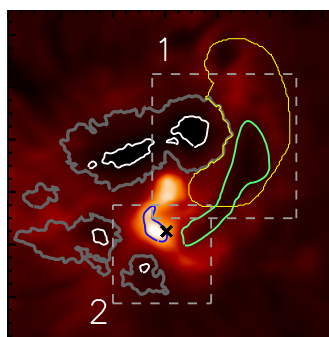

10:36:10 UT

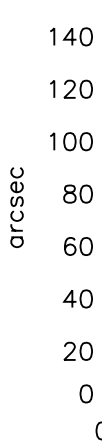

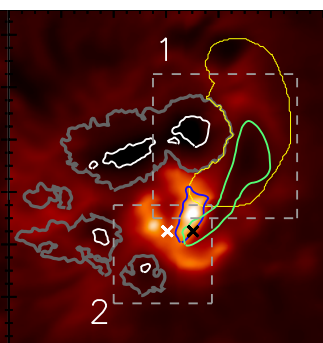

10:39:10 UT

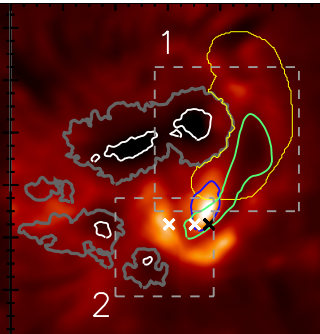

10:42:10 UT
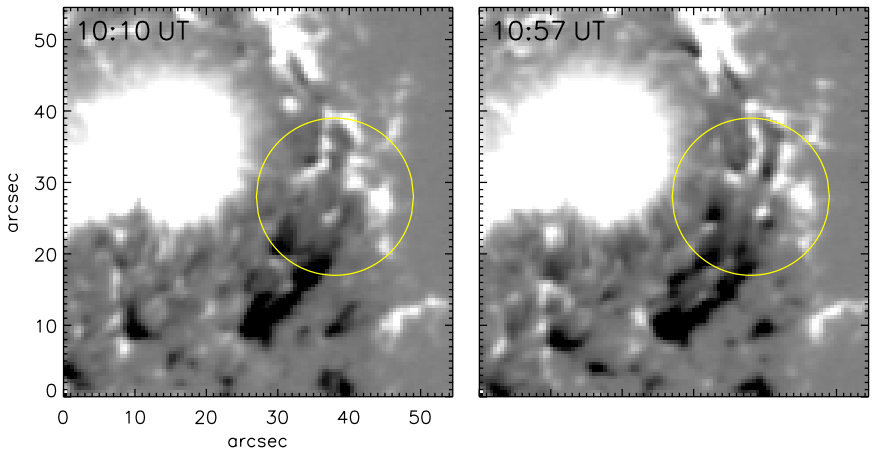

Fig. 7. Events of flux emergence at the neutral line close to the leading sunspot. The field-of-view in the figure corresponds to the region marked by the grey dashed square labelled "1" in Fig. 6. The yellow circle encloses patches of negative polarity that emerge close to the time of the M5.6 flare.

was estimated to be $-1.9 \times 10^{18} \mathrm{Mx}$, which steadily approached zero at 10:34 UT when it was no longer visible.

Figure 10 shows the temporal evolution of magnetic flux in the two small patches described above. The positive and negative fluxes are depicted, and their values correspond to the violet $y$-axis on the right. As described above, there is an abrupt reduction of flux in the positive polarity patch close to the time of the C2.9 flare.

The flux of the previously emerged negative-flux region outlined by white contours in the first column of Fig. 9 is shown in Fig. 10. Here the relative magnetic flux was calculated with respect to its value at 10:52 UT at the peak of the M5.6 flare $\left(F_{\mathrm{n}}(t)-F_{\mathrm{n}, \text { ref }}\right.$, where "ref" indicates the peak time of the flare and $F_{\text {n,ref }}=-2.0 \times 10^{20} \mathrm{Mx}$ ). Starting at 10:30 UT, the relative flux steeply decreases until 10:49 UT, i.e. until shortly after the M5.6 flare onset. After this point, the flux first increases again to a value of $-2.1 \times 10^{19} \mathrm{Mx}$ at 11:06 UT, but in the long run much of it cancels out (Fig. 2, panels P12-P15). To compare this reduction in negative flux prior to the M5.6 flare, we also determined the positive flux in the satellite spot (within the field-of-view displayed in Fig. 6). In this case, the relative flux is calculated as before, namely, $F_{\mathrm{p}}(t)-F_{\mathrm{p} \text {, ref, where }}$

Fig. 6. Evolution of the C2.9 precursor flare towards the onset of the M5.6 flare. Each ChroTel $\mathrm{H} \alpha$ image has been individually scaled to its maximum intensity. The green and blue contours outline the filament and the flare brightenings, respectively, as observed at $\mathrm{H} \alpha$. The white and grey contours correspond to the sunspot umbra-penumbra and penumbra-quiet Sun boundary, respectively. The yellow contour shows the mask used in deriving the amount of flux emerging near the neutral line, plotted in Fig. 8. The black and white crosses indicate the position of the leading edge of the brightness front in the current and previous frames, respectively. The dashed grey squares labelled 1 and 2 are small regions selected for analysing magnetic cancellation and/or emergence events.

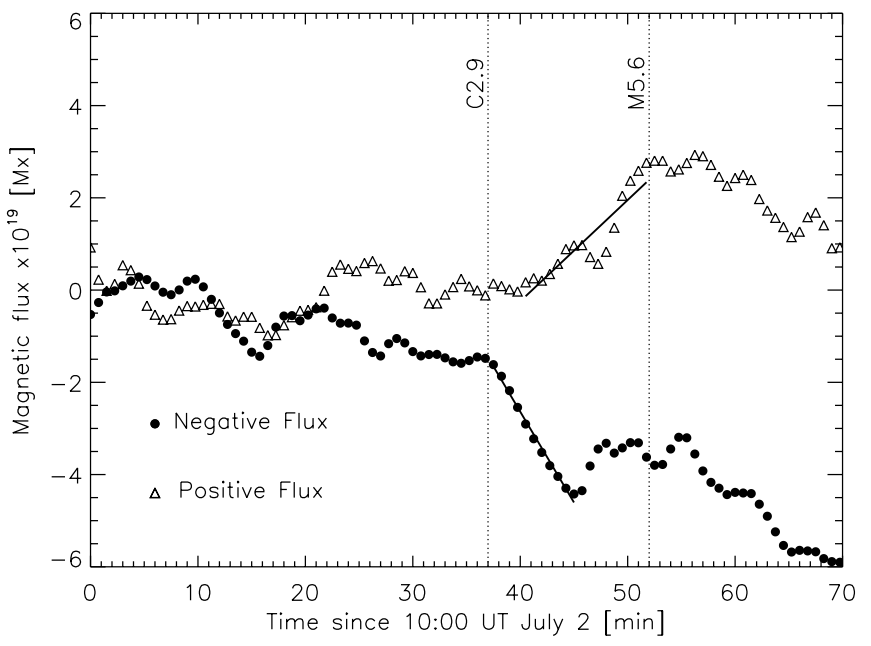

Fig. 8. Temporal change of magnetic flux within in the area enclosed by the yellow line in Fig. 6, including linear fits to the steepest parts.

$F_{\mathrm{p}, \text { ref }}=5.1 \times 10^{20} \mathrm{Mx}$. The positive flux exhibits fluctuations around a nearly stationary average value rather than a systematic change with time. The fluctuations include minor peaks at the times of the C2.9 and M5.6 flares, but the peak values are not correlated with the magnitudes of the flares. Similar to the negative flux patch next to it, the flux in the satellite spot is determined by both continuing emergence and cancellation, resulting in the dominance of fluctuations on the time scale of the plot. On a longer time scale, the satellite spot is dominated by continuing emergence (Fig. 2).

\subsection{Properties of the runaway sunspot}

We follow the runaway part of the leading sunspot after the splitting by tracking its centroid position. Furthermore, we fit an ellipse to the umbra-penumbra boundary and retrieve its centre, major and minor axes, and its rotation. Using the above parameters, one finds that the runaway sunspot drifts about $43 \mathrm{Mm}$ from its initial position in a duration of $60 \mathrm{~h}$ after the flare, moving with a nearly constant speed of about $210 \mathrm{~m} \mathrm{~s}^{-1}$ (top panel of Fig. 11). The sunspot also exhibits significant rotation. 


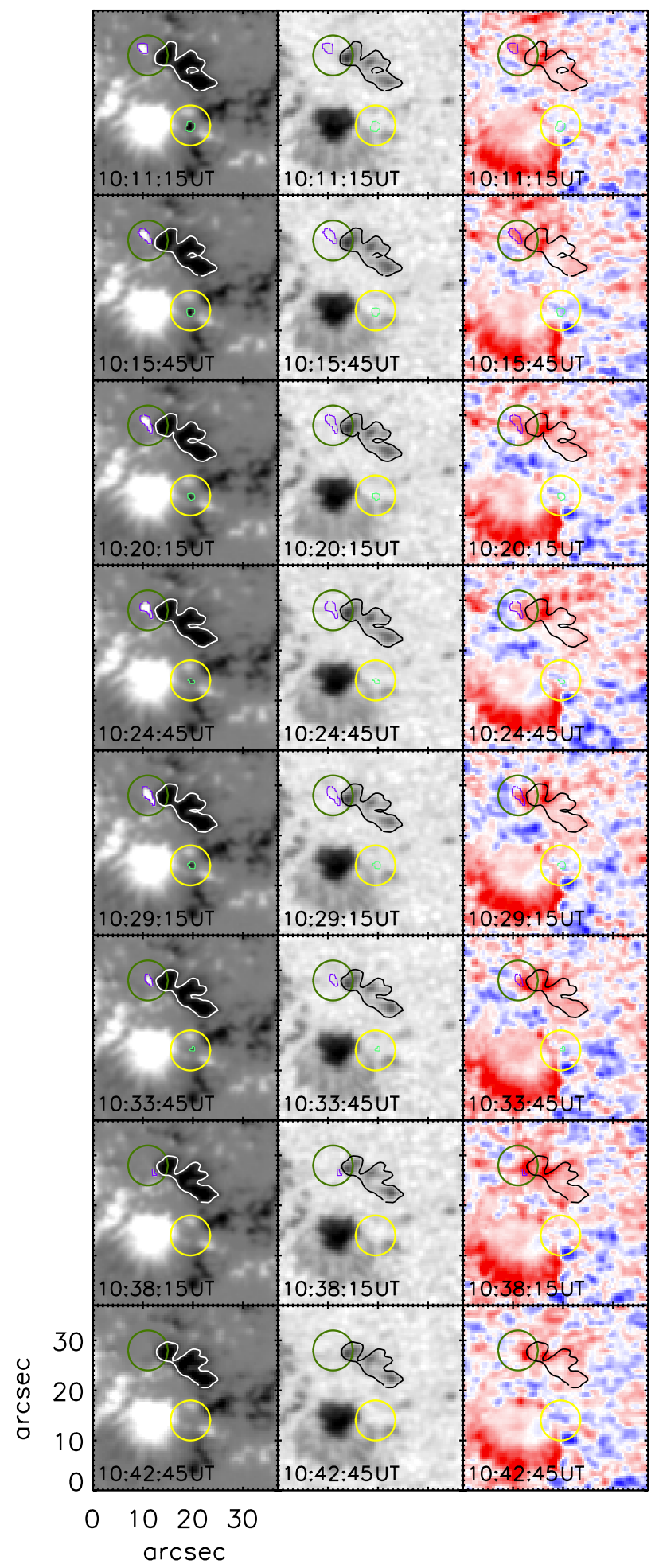

Fig. 9. Photospheric magnetic flux cancellation/disappearance near the polarity inversion line. The images of the time sequence correspond to the LOS magnetograms (left), continuum intensity (middle), and dopplergrams (right) for the region marked by the dashed grey square labelled "2" in Fig. 6. The green (yellow) circle encloses a positive (negative) magnetic patch outlined in violet (turquoise). The magnetograms, continuum intensity, and dopplergrams have been scaled between $\pm 1000 \mathrm{G}, 0.1-1.05 I_{\mathrm{QS}}$, and $\pm 1.5 \mathrm{~km} \mathrm{~s}^{-1}$, respectively. The violet and turquoise contours enclose fields greater than 80 and $50 \mathrm{G}$, respectively, and have been enhanced by a factor of six for clarity. The white contour in the left column (black in Cols. 2-3) outlines a region of previous flux emergence where the flux also decreases in the time before the flares.

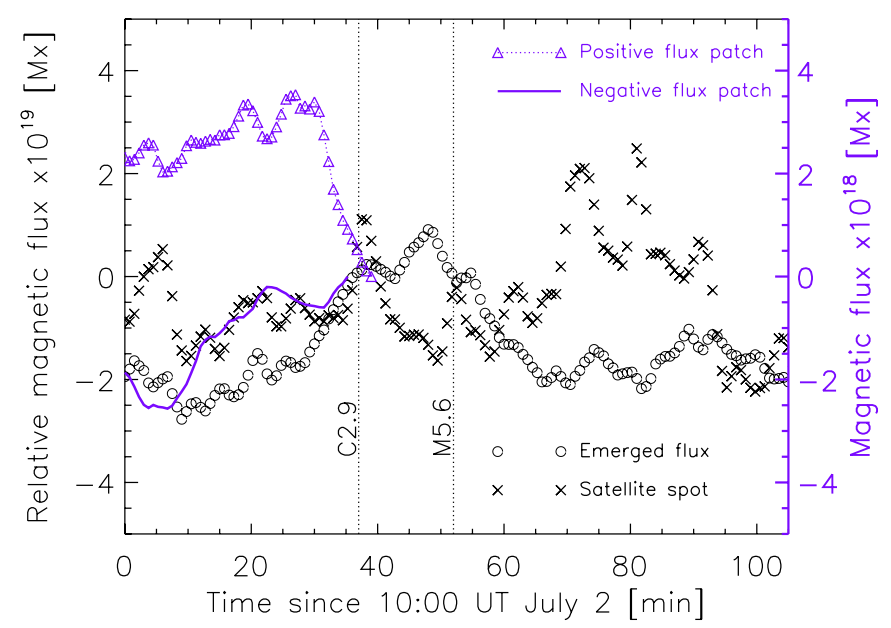

Fig. 10. Temporal evolution of magnetic flux. The magnetic flux of the positive and negative polarity patches identified in Fig. 9 is shown as dotted lines with violet triangles and thick violet lines, respectively. Their scaling is shown on the violet $y$-axis to the right. The relative magnetic flux of the previously emerged negative-polarity region near the new satellite sunspot is shown as open circles. Black crosses show the positive flux in the satellite spot in the area shown in Fig. 6. The last two quantities are referenced to their values at 10:52 UT.

The rotation angle is measured with respect to solar west (positive $x$-axis) and increases counter-clockwise. The bottom panel of Fig. 11 shows that the sunspot rotates by nearly $70^{\circ}$ in the course of $60 \mathrm{~h}$ with a maximum angular speed of $4.9^{\circ} \mathrm{h}^{-1}$ on July 3 , which was estimated from a linear fit to the steepest part of the plot. This equals the highest rotation rate of a sunspot found so far (Zhang et al. 2008a). Following Brown et al. (2003), the linear speed in the outer penumbra, $20^{\prime \prime}$ from the centre of the sunspot, is about $360 \mathrm{~m} \mathrm{~s}^{-1}$. By comparison, the other half of the leading sunspot appears to be fairly stationary and does not exhibit any discernible rotation.

Overall, we find a complex pattern consisting of flux increase (emergence) at the northern end of the filament and simultaneous increase and decrease in the flux (including cancellation) in several patches near the southern end of the filament. Changes of the order of $\pm(2-4) \times 10^{19} \mathrm{Mx}$ occur within $30 \mathrm{~min}$ prior to the eruption. These are embedded in longer trends of flux change associated with flows converging towards the neutral line both in the bow-shaped arc ahead of the leading sunspot and in the part adjacent to the satellite polarity.

Using coronal loops to trace the basic magnetic structure of the region, we find that the two fragments of the splitting spot possess completely different connections to the ambient flux. Figure 12 and the accompanying animation ${ }^{2}$ display AIA $171 \AA$ images before and after the flares. The loops in the AIA images were identified using the procedure described by Aschwanden (2010). The routine, originally developed for Transition Region and Coronal Explorer (TRACE, Handy et al. 1999) images works equally well for the AIA $171 \AA$ Amages with appropriate adjustments of a few input parameters ${ }^{3}$. The loops in the AIA images, identified automatically and by hand, have been overlaid on the photospheric LOS magnetograms. These images show that all coronal loops rooted in the rear part of the leading sunspot connect to the following negative polarity

\footnotetext{
2 Available as online material.

3 The code is available at http://www.lmsal.com/ aschwand/ software/ and is also included in the Solar Soft analysis suite under
} \$SSW/packages/mjastereo/idl/ 

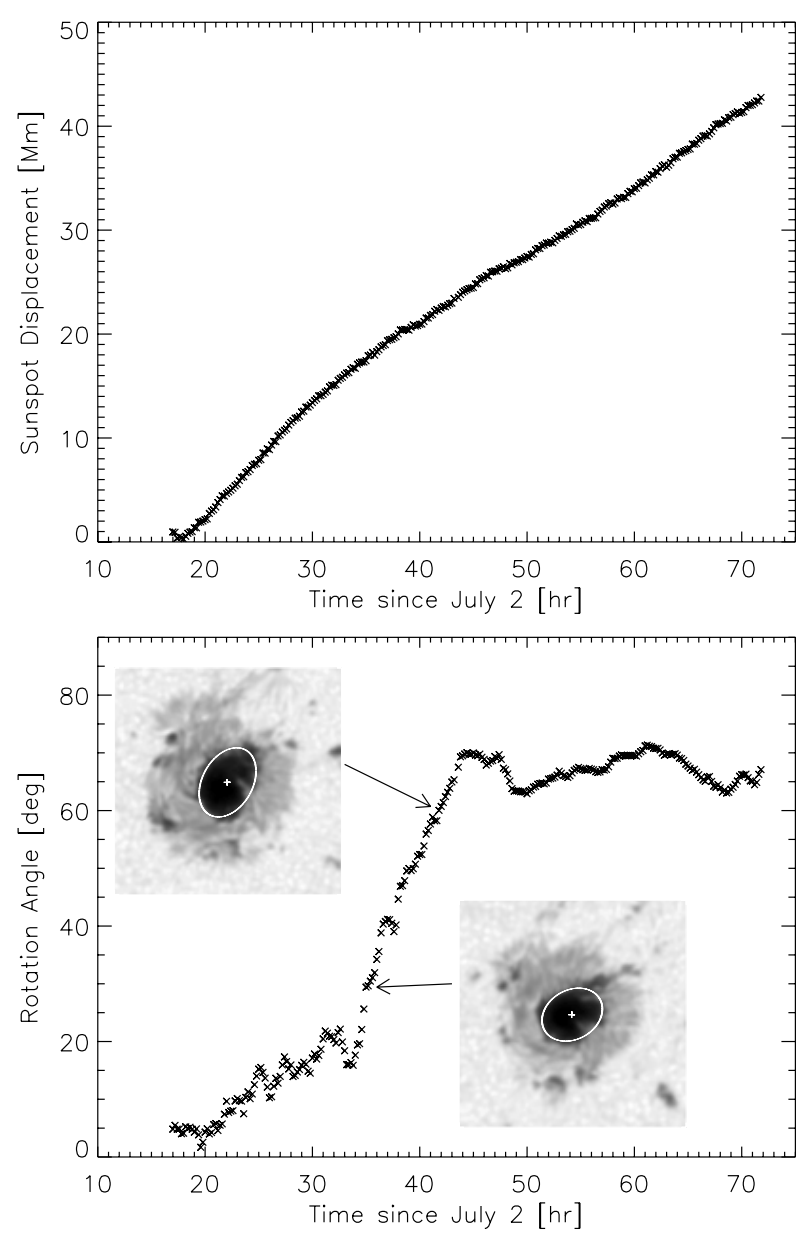

Fig. 11. Displacement and rotation of the runaway sunspot are shown in the top and bottom panels, respectively, after separation from its host area around 16:00 UT on July 2. In the bottom panel the continuum images at two different instances along the steep part of the plot are shown. The thin white lines mark the umbra-penumbra boundary retrieved from fitting an ellipse to the observed contour and the white pluses represent the centre positions of the ellipses.

of AR 11515, while all loops rooted in the front part, although diffuse and fainter, clearly connect to the west side (blue loops in panels 1 and 2 of Fig. 12) where the diffuse trailing negative polarity of AR 11514 and enhanced negative network flux south of AR 11514 are located. The existence of the latter connections is confirmed by the development of a distinct ribbon-like brightening in this area in the course of the M5.6 flare (panel 4). A new set of bright loops and enhanced diffuse brightness, which are obvious after the rising filament has largely faded (panel 4), extend from the ribbon to the leading sunspot and the arc-shaped neutral line, demonstrating that these structures are magnetically connected. The lack of loop connectivity between the rear half of the leading sunspot and the negative flux in and around AR 11514 is furthermore evident from the blue loops in panel 3 of the figure. The panel shows that the longer blue loop connects to a positive flux patch north of the leading sunspot while the shorter blue loop points to the middle of the splitting sunspot (not to its rear half).

The AIA images in the course of the flare also show that the great majority of the loops connecting the rear part of the splitting sunspot with the trailing negative flux of the active region do not experience any change (apart from a brief oscillation of the southernmost ones); see the animation accompanying Fig. 12. Only few new bright loops become visible, presumably located in the interface to the overlying flux. This substantiates the conclusion that the two parts of the splitting sunspot have different magnetic connections.

\subsection{Shear flows around the runaway spot}

As discussed in Sect. 3.3, the splitting of the sunspot involves a shear flow component relative to the neutral line under the northern end of the filament in addition to the approach to the neutral line. No shear flows are seen within the splitting spot before and around the time of the flare. This changes on July 3, when the runaway sunspot approaches the opposite polarity patches closely. We infer the shear flows using local correlation tracking (LCT, November 1986; November \& Simon 1988; Welsch et al. 2004; Fisher \& Welsch 2008). After several trials in selecting the parameters of the LCT technique, an apodizing window having a width of $4^{\prime \prime}$ and a time difference of 12 min between the images were chosen. Three successive velocity images were averaged to reduce the noise in the measurements.

Using the parameters of the ellipse that traces the umbral boundary (Sect. 3.4), we construct 15 equally spaced azimuthal contours starting from the umbra-penumbra boundary and progressively displaced radially outward. Zero azimuth $\phi$ on the elliptical contour starts at the positive major axis and increases in the counter-clockwise direction. This is illustrated in Fig. 13, where the equidistant contours have been overlaid on the LOS magnetogram and the horizontal flow vectors. The value of the azimuth is given in the adjacent colour bar. As the runaway sunspot rotates, the contours rotate along with it, since the tilt of the ellipse is known. We restrict our analysis to $0^{\circ} \leq \phi \leq 130^{\circ}$ and $230^{\circ} \leq \phi \leq 360^{\circ}$ and calculate the shear angle $\phi_{\mathrm{s}}$ on the contours as the one made between the flow vector and the normal to the contour. The value of $\phi_{\mathrm{s}}$ can vary from $0-180^{\circ}$, where $0^{\circ} \leq \phi_{\mathrm{s}} \leq 90^{\circ}$ and $90^{\circ}<\phi_{\mathrm{s}} \leq 180^{\circ}$ correspond to outward and inward flows, respectively.

The top panel of Fig. 14 shows the temporal evolution of the shear angle, averaged over the azimuth, for the eight outermost contours. The corresponding mean horizontal velocity is plotted in the middle panel. A representative azimuth position of strong shear flows, given in the bottom panel, is obtained by averaging the azimuth values of all pixels on the contour where $30^{\circ} \leq \phi_{\mathrm{s}} \leq 90^{\circ}$.

Although one does not observe a strong time dependence in the shear flow, large shear flows $\left(\phi_{\mathrm{s}}>60^{\circ}\right)$ are predominantly seen on July 3 . The mean shear angle in the outer penumbra of the runaway sunspot is about $45^{\circ}$, while the horizontal speed ranges from to $220-500 \mathrm{~m} \mathrm{~s}^{-1}$ with mean values of about $325 \mathrm{~m} \mathrm{~s}^{-1}$. In addition, large shear angles are not associated with strong flow speeds, which is evident for two instances on July 3 where the speeds are about $300 \mathrm{~m} \mathrm{~s}^{-1}$. The bottom panel of Fig. 11 indicates that the shear flows span a large section of the sunspot's circumference during the course of two days and are not confined to a specific range of azimuths. There is, however, an antisymmetric trend around $0^{\circ}$ azimuth wherein the shear flows change azimuth from $100^{\circ}$ to $-80^{\circ}$ close to the end of July 3. The nature of the shear flows is also reflected, however weakly, in the relative azimuthal lag between the inner and outermost contours. This can be seen towards the end of July 2 and in the early parts of July 3 and July 4.

\section{Discussion}

\subsection{Triggering of the flare}

The considered event exemplifies the possible complexity in the evolution towards solar eruptions. It reveals all three ba- 

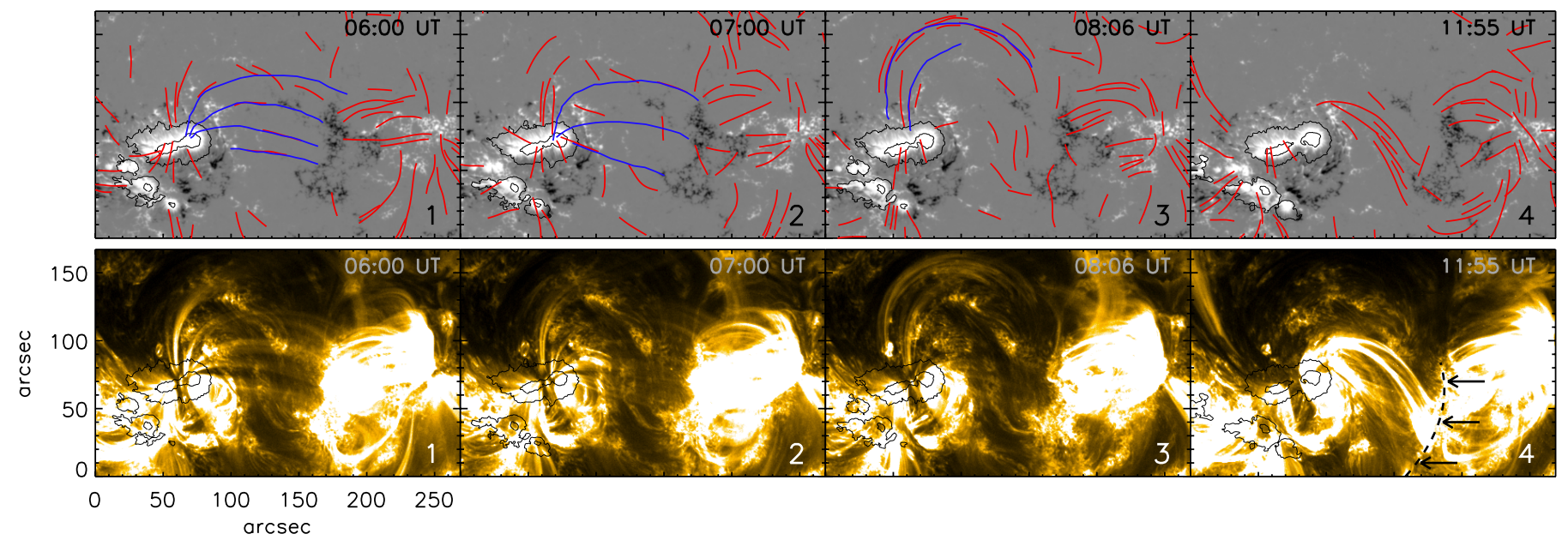

Fig. 12. (Movie online) Evolution of coronal loops in AR 11515. The top and bottom panels show HMI LOS magnetograms and AIA $171 \AA \AA$ images, respectively. The loops identified in the AIA images using the automated identification routine have been overlaid on the magnetograms and are shown in red. The blue loops represent those that were traced manually. The black contours correspond to the continuum intensity and outline the sunspots in the active region. The dashed line and arrows in panel 4 correspond to a distinct ribbon-like brightening in the course of the M5.6 flare. The temporal evalution of the coronal emission in the lower panels is also available as an animation in the online edition.

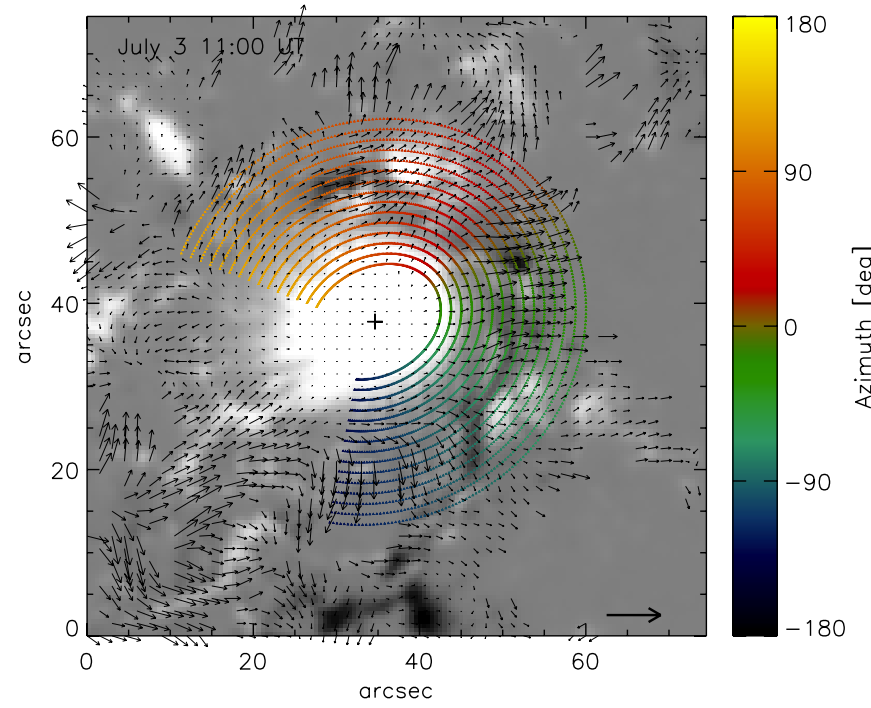

Fig. 13. Horizontal flow field in the runaway sunspot (relative to its centre). The arrows indicate the flow vectors derived from LCT, which have been overlaid on the LOS magnetogram. The thick black horizontal arrow at the bottom right corner corresponds to $1 \mathrm{~km} \mathrm{~s}^{-1}$. The equidistant elliptical contours span an azimuth range of $-130^{\circ}$ to $130^{\circ}$, which is scaled according to the vertical colour bar. A value of $0^{\circ}$ azimuth coincides with the positive semi-major axis and increases in counterclockwise direction. The plus corresponds to the centre of the sunspot.

sic photospheric driving processes to be at work, includes a precursor flare, and additionally involves a sunspot that splits in an unusual manner. Given this complexity, it is not clear whether a dominant causal relationship between one of the driving processes and the eruption can be isolated, and we refrain from stretching the interpretation of the data that far. Rather, we discuss how the various processes may contribute to the destabilization of the coronal magnetic field observed as the filament eruption, M-class flare, and CME.

The two major photospheric changes prior to the eruption are the splitting of the active region's leading sunspot and the emergence of flux that forms a group of satellite sunspots and a new neutral line ahead of the leading sunspot and the satellite spots. The erupting filament forms at this neutral line.

While the splitting of the leading spot and subsequent separation of its front half represent the major change of the region in white light, they may have only a secondary effect on its largescale magnetic topology. This is suggested by the fact that the two fragments have completely different connections to the ambient photospheric flux sources, indicating that the separation is not favorable for a topology change (the flux of the front half of the leading sunspot connects westward while the flux of its rear half connects eastward). Thus, it is possible (although not proven by our analysis) that the splitting of the spot neither changes the large-scale topology nor enforces the approach and reconnection of flux within the central part of the active region. (Verifying this conjecture would require sophisticated modelling of the evolving coronal field comprising two active regions, which is beyond the scope of the present paper.)

However, the splitting causes the approach of the separating front half to the new arc-shaped neutral line formed by emerging flux ahead of it. Seen from the neutral line, this is equivalent to a long-lasting inflow. Such inflow is crucial for the formation and destabilization of a coronal flux rope above the neutral line by magnetic reconnection if the field above the neutral line is sheared (van Ballegooijen \& Martens 1989; Inhester et al. 1992; Amari et al. 2011). The shearing is realized by the northward component of motion of the front half. In decaying active regions, inflows towards the neutral line are always associated with flux cancellation, but here flux emergence is dominant and prevents the presumably embedded cancellation events of smallscale flux patches (Fig. 7) from showing up clearly in the period prior to the flare. The splitting spot enforces the flows in a coherent manner over many hours, and the resulting reconnection continually adds flux to the newly formed rope. Increasing flux is known to eventually destabilize a flux rope (e.g., Mackay \& van Ballegooijen 2006; Su et al. 2011; Kliem et al. 2013).

A basically similar situation of long-lasting inflows and associated flux cancellation develops at the southern part of the neutral line adjacent to the satellite spots. Thus, the flows converging at the neutral line ahead of the leading spot, and at the satellite spots, are a prime candidate for the photospheric driver that causes the formation of a coronal flux rope and its subsequent 

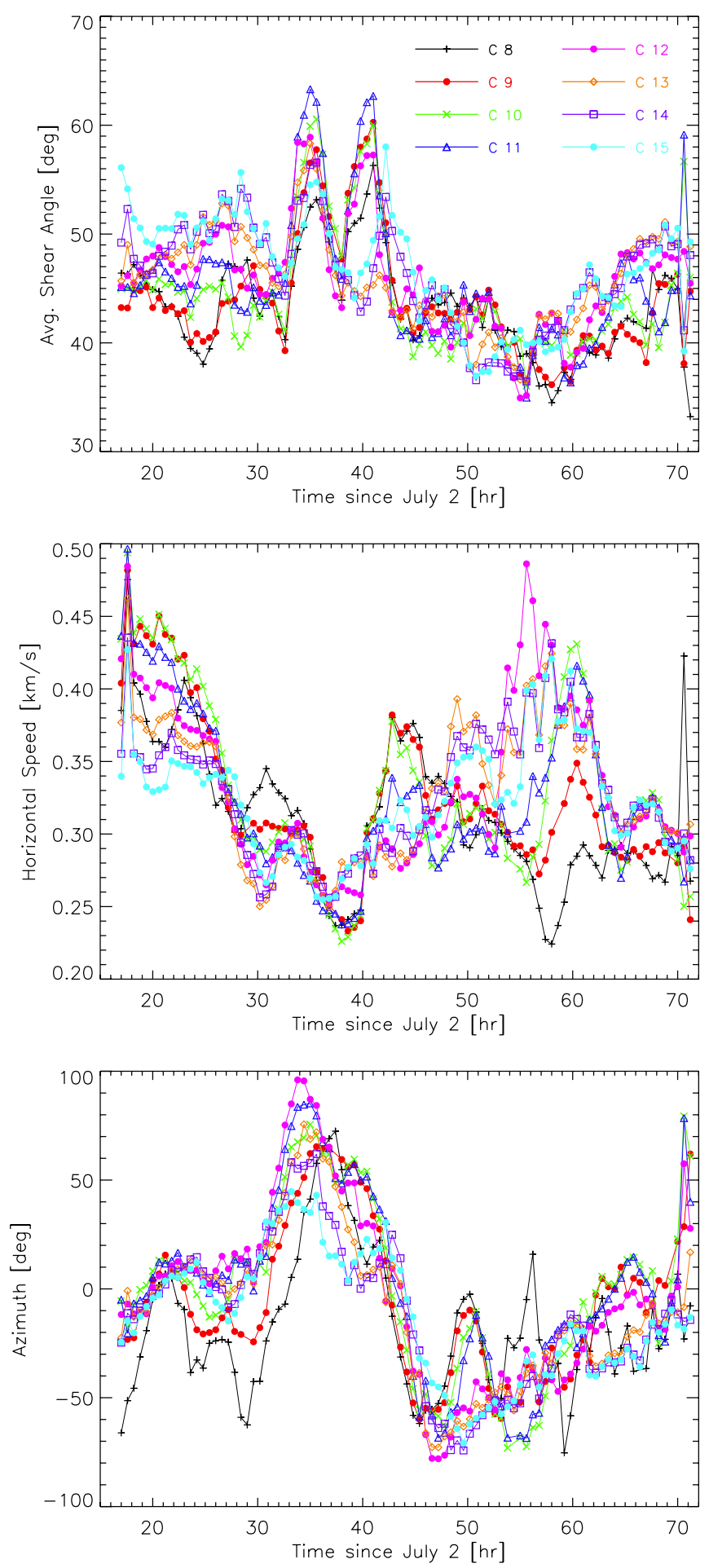

Fig. 14. Temporal evolution of shear flow around the runaway sunspot. Top to bottom: shear angle, horizontal flow speed, and azimuth. The azimuthally averaged values for the different equidistant radial contours are depicted in colour, and the legend is provided in the top panel.

destabilization. The splitting of the leading spot is a major contributor to these flows, at least in the northern part of the area.

The other important candidate process is flux emergence (Sect. 1). In the considered event, most of the flux ahead of the splitting spot emerges in the period of one to two days immediately before the eruption, thus forming the neutral line where the eruption originates. Generally, flux emergence can cause or support eruptions in five ways: (1) by reconnection with a preexisting flux rope, enhancing its flux; (2) by reconnection with the ambient flux of a pre-existing flux rope, reducing the stabilizing effect of the ambient flux (Chen \& Shibata 2000); (3) by reconnection with a pre-existing arcade field above the neutral line, thus forming and destabilizing a coronal flux rope (Kusano et al. 2012); (4) by fully emerging a new flux rope into the corona (e.g., Low 1996); and (5) by partially emerging a flux rope into the corona and transforming the emerged part (by reconnection) into a new flux rope (e.g., Manchester et al. 2004; Archontis \& Hood 2010). The observations of the event are fully consistent with the first two options, which both represent the destabilization of an existing flux rope, that was formed by reconnection, which in our case, was mainly driven by the moving front half of the splitting spot. This holds true for the flux emergence in the arc-shaped part of the neutral line ahead of the splitting spot (i.e., under the main body of the filament), as well as for the emergence of the satellite sunspots and the negative flux near them (i.e., under the southern end of the filament, where the eruption starts). The third option leaves the occurrence of the precursor flare unexplained. The fourth implies a systematic separation pattern of opposite-polarity flux on the sides of the neutral line as a dominant signature in the magnetogram (MacTaggart \& Hood 2010), which is not apparent in our case. The fifth is associated with strong shear flows at the neutral line, intrinsic to flux emergence. However, the shear flows in the present event are externally driven by the splitting spot. Additionally, that a substantial part of the emerging flux consists of mixed small-scale polarities does not support options four and five.

Finally, the very close spatial and temporal association of the weaker precursor flare with the eruptive M-class flare and, especially, the signs of interaction of its propagating emission front with the filament exactly at the onset time of the eruption are highly suggestive of a causal relationship. Since a propagating emission front cannot form an unstable magnetic field configuration in such a short time span, the precursor flare must play the role of an external trigger (a "final drop") acting on a configuration already close to the onset of instability.

\subsection{Splitting of the sunspot}

The manner and duration of the observed sunspot splitting is quite distinct from what is typically seen during the breakup of sunspots that involves one or more light bridges (Garcia de La Rosa 1987). The formation of light bridges is taken as evidence for convective penetration that disrupts the dynamic and mechanical equilibrium of a sunspot. Louis et al. (2012) presented an example of a regular, isolated sunspot splitting in this manner, and they found that the splitting required about $48 \mathrm{hr}$ following the formation of a light bridge, although only a small part of the sunspot broke away. The splitting of the leading sunspot in this investigation is initiated by a stretching instead of light bridge formation, and proceeds within about a day, so much faster than usual. While the front half separates from the active region at a considerable speed of $210 \mathrm{~m} \mathrm{~s}^{-1}$ (consistent with the values reported in Herdiwijaya et al. 1997) and shows strong rotation, as well as considerable shear flows, the rear half stays essentially stationary within the active region without any signs of rotation or shear flows. The rotation rate of the runaway sunspot equals the highest rotation rate of a single sunspot found previously (compare, e.g., with Brown et al. 2003; Zhang et al. 2008a; Yan et al. 2012); thus, it is a further unusual property of the splitting process in the considered event. 
A hint at the cause of the non-standard sunspot breakup process may be found in the magnetic structure of the region. We have noted in Sect. 3.4 that the two fragments possess completely different magnetic connections. Essentially all flux of the separating front fragment connects to the neighbouring AR 11514 west of AR 11515 or to network flux in the vicinity of AR 11514. The rear fragment is apparently completely connected within AR 11515. Thus, we consider the possibility that the front fragment originates in the same flux system deep below the photosphere (or from the same rising Omega loop) as AR 11514 and becomes temporarily trapped within AR 11515 through a merger with the leading spot of that region, which has the same polarity. The splitting of the spot could in this case result from magnetic tension forces still acting on the front half. The action of such forces is also suggested by the stretching of the spot prior to the splitting.

The alternative possibility of tension forces provided by the coronal connections to AR 11514 is unlikely for energetic reasons, as detailed below, and it could not cause the rotation because the coronal loops do not indicate the presence of significant twist. We therefore interpret the breakup and further separation of the front fragment as an attempt to merge with the active region it is magnetically connected to. The approach to AR 11514 is seen to continue throughout the region's rotation to the western limb. This scenario naturally explains the rotation of the separating fragment as the continued rise of a flux tube that is still connected to its source deep in the solar interior (Brown et al. 2003; van Driel-Gesztelyi \& Petrovay 1990; Leka et al. 1994) after the rise was temporarily stalled by the connection with the leading spot of AR 11515. It is also in line with the high rotation rate. The counter-clockwise rotation then implies a left-handed twist, and this sense of the twist is indeed indicated by the clockwise whirl of several coronal loops connected to the fragment (Fig. 12) and by the reverse S-shaped bending of the filament's northern end (Figs. 3, 5, and 12).

We also note that the splitting of the sunspot begins only a few hours after the nearby emergence of the satellite sunspots, which subsequently merge with the rear half. It is obvious that this interaction represents a major perturbation of the spot, and it is likely that it supports the onset of its splitting, especially if the spot is indeed in a metastable equilibrium of two differently rooted and connected parts.

The suggested interpretation implies that at least the front fragment of the splitting spot had not experienced a disconnection from its roots deep in the convection zone. The general occurrence of such a disconnection is suggested by a change in the dynamical properties of spots from more active to more passive evolution (with regard to the ambient convection) soon after their emergence (Schrijver \& Title 1999); however, this is still an unresolved issue. Numerical modelling by Schüssler \& Rempel (2005) shows dynamical disconnection at depths of 2-6 Mm and within about three days from the formation of a spot. More advanced modelling (Rempel 2011b) instead indicates that the process would severely undermine the stability of the spot, if working on these scales. Therefore, dynamical disconnection should proceed only on time scales comparable to, or longer than, the time the spot remains coherent, and at correspondingly greater depths (e.g., at about 30-50 Mm for spot life times of a week). This is supported by the observational study of Švanda et al. (2009), who found clear signatures of disconnection only in about a third of the investigated sunspots, with inferred disconnection depths in the range $\sim 5-50 \mathrm{Mm}$. Our interpretation of the sunspot splitting in AR 11515 is consistent with this more recent picture.
Finally, we compare the kinetic energy associated with the splitting of the sunspot with the energy released by the flare. The kinetic energy can be calculated as $E_{\text {kin }}=0.5 A \mathrm{~d} \rho v_{\text {split }}^{2}$, where $A$ is the area of a cylindrical flux tube, $d$ the depth, $\rho$ the density, and $v_{\text {split }}$ the splitting speed of the sunspot. However, assuming that the proper motion of the runaway sunspot fragment at the surface is due to the vertical ascent of an inclined flux tube, not to the horizontal displacement of the tube, $v_{\text {split }}$ can be replaced by $v_{\text {rise }}$, where $v_{\text {rise }}=v_{\text {split }} \tan \theta$, and $\theta$ is the inclination of the tube with respect to the surface. Since the considered volume is strongly stratified, the area of the tube as a function of depth $z$ can be approximated as $A(z)=A_{\mathrm{s}} \exp \left(-z / \tau_{B}\right)$, following flux conservation, and the density can be approximated as $\rho(z)=\rho_{\mathrm{s}} \exp \left(z / \tau_{\rho}\right)$. The subscript 's' refers to the value at the photosphere, while $\tau_{B}$ and $\tau_{\rho}$ correspond to the scale heights of the magnetic field strength and the density, respectively. The kinetic energy can then be estimated as the integral $\int_{0}^{d} 0.5 A(z) \rho(z) v_{\text {rise }}^{2} \mathrm{~d} z$. Assuming $v_{\text {rise }}$ is $500 \mathrm{~m} \mathrm{~s}^{-1}$, $\rho_{\mathrm{s}} 10^{-7} \mathrm{~g} \mathrm{~cm}^{-3}, \tau_{B} 2000 \mathrm{~km}$ (Murphy 1990; Rempel et al. 2009), and $\tau_{\rho} 500 \mathrm{~km}$ (Rempel 2011a), a conservative estimate of the vertical depth $d$ of $6 \mathrm{Mm}$ (following Schüssler \& Rempel 2005), and using the observed spot radius of $144^{\prime \prime} 5$ at the surface, we obtain a value of $2 \times 10^{32} \mathrm{erg}$ for the kinetic energy. This is greater than the thermal energy of the soft X-ray emitting plasma and the bolometric irradiance, which are typically of the order of $10^{30} \mathrm{erg}$ and $10^{31} \mathrm{erg}$, respectively, for M-class flares (Emslie et al. 2012). This also quantitatively validates, at least to a firstorder approximation, that the splitting of the sunspot is not the consequence but a possible driver of the flare. If disconnection happened at higher values of $d$, or if the runaway spot was indeed still rooted deep in the convection zone, the kinetic energy associated with the splitting would be much higher.

A similar conclusion can be drawn regarding the relevance of the coronal flux connecting to AR 11514 for the sunspot splitting process. Such extended high-lying coronal flux is usually relatively close to the potential-field state, so it contains much less free energy than the highly sheared core field of a flare-productive active region, which powers the eruptions, as in the considered event. Therefore, these loops could not store the $>10^{32}$ erg of free energy required to drag the front fragment of the sunspot away from AR 11515.

Our estimate of the relation between the energy released in the considered M-class flare and the kinetic energy of the associated sunspot motion is the opposite of the result for an X-class flare analysed in Anwar et al. (1993). In that case, the whole sunspot closest to the flare position reached an apparent velocity of about $2 \mathrm{~km} \mathrm{~s}^{-1}$, but only for at most $430 \mathrm{~s}$. From this duration, the maximum depth of the motion was estimated to be only about $1.2 \mathrm{Mm}$, based on the Afvénic propagation of stresses at the photospheric level (caused by the flare) into the solar interior. Indeed, it is highly unlikely that a subphotospheric flux tube extending over many megameters would be accelerated and decelerated within the observed short time span of the sunspot displacement. From the much smaller depth of the motions involved, a much lower energy of $<10^{30} \mathrm{erg}$ was estimated. Thus, the very different time scales and implied different spatial scales of the two events of flare-associated sunspot changes allow for all these different conclusion.

\section{Conclusions}

This investigation of a splitting sunspot, the subsequent separation of the leading fragment from the active region, and 
the associated eruption involving a filament, M-class flare, and $\mathrm{CME}$ yields the following conclusions. A complex interplay of photospheric and coronal processes was involved in the evolution of the region to the eruption. The splitting of the sunspot has likely played a major role by enforcing a coherent, long-lasting inflow and shear flow at the photospheric neutral line ahead of the spot. This could have resulted in the formation of a coronal flux rope that held the filament and the eventual destabilization of the rope. The emergence of flux created the neutral line in the first place; however, we did not find any indications of flux rope formation directly caused by the emergence. Rather, the continued emergence has likely contributed to the destabilization of the flux rope. Associated with the formation of satellite sunspots, the emergence was strongest in the southern part of the neutral line under the filament, where the eruption started. Additionally, converging flows and resulting flux cancellation occurred at this part of the neutral line and have likely also contributed to flux rope formation and destabilization. A C-class precursor flare, possessing close spatial and temporal associations with the M-class flare and showing signs of interaction with the filament at the onset time of the eruption, has likely played the role of a "final drop" acting on an equilibrium already very close to the onset of instability.

The splitting of the sunspot showed the following unusual characteristics: it began by stretching instead of light bridge formation, proceeded relatively fast, and set free a sunspot fragment that rotated rapidly in the course of its separation from the remaining spot, which was essentially stationary. The separation of the fragment from the active region was rather fast and large, although not unusual. Supported by the different magnetic connections of the fragments, seen as coronal loops, these properties suggest that the fragments may have originated in different flux systems, or Omega loops, rooted deep in the solar interior, and that the front fragment joined the flux of the active region only temporarily through its merging with the other flux in the leading sunspot. The perturbation of the leading spot by the emergence of satellite polarities shortly before and during the splitting, as well as magnetic tension forces by the subphotospheric flux connections of the leading part, are the factors of potential relevance for the splitting. The latter can explain the stretching of the sunspot and the strong separation of the front fragment, which let the fragment approach the preceding active region where its coronal magnetic connections were anchored. The standard interpretation of the fragment's counter-clockwise rotation as the continued rise of a twisted subphotospheric flux bundle (after an interruption due to trapping in the sunspot) is supported by the clockwise whirl of some coronal structures in the vicinity of the fragment, which indicate a left-handed twist. This interpretation of the sunspot splitting implies that at least the flux of the separating fragment was still connected to its roots deep in the convection zone.

The eruption mechanism usually associated with flux dispersal and cancellation in decaying active regions gains relevance here in a phase of major change and additional flux emergence, owing to the motion of the split sunspot fragment towards a neighbouring neutral line. Previous observations of splitting sunspots found flaring associated with a very similar driving, often including a major flare (Wang et al. 1991; Schmieder et al. 1994; Denker \& Wang 1998). A similar situation occurs when sunspots of different active regions, or within complex regions, approach each other, typically also causing major eruptions (e.g., Kovacs \& Dezso 1986; Kubo et al. 2007; Sun et al. 2012). Thus, events like the one studied here may serve as testbeds for detailed numerical studies of this important eruption mechanism under relatively well defined observational constraints. In addition to the comprehensive observational coverage provided by current instruments, the measurements of the magnetic and velocity fields are relatively reliable, thanks to their coherent and high values. This may also allow checking the key conjecture of this mechanism - that a flux rope forms prior to the eruption - against the opposing view of eruption onset in a sufficiently sheared arcade (Karpen et al. 2012).

Multi-wavelength observations with good spatial and temporal resolution, including spectroscopy and polarimetry in the photosphere and chromosphere, have proven to be crucial in this study for resolving the complex photospheric dynamics and for at least partially disentangling their equally complex relationships to the resulting coronal evolution. These diagnostic tools provide important information on the nature and dynamics of plasma and magnetic fields at the site of the activity. It is anticipated that new instruments such as the GREGOR Fabry Pérot Interferometer (GFPI, Puschmann et al. 2012a,b, and references therein), the BLue Imaging Solar Spectrometer (BLISS, Denker 2010; Puschmann et al. 2012a, 2013), and the Chromospheric Magnetometer (ChroMag, Bethge et al. 2012b) will be pivotal for carrying out such investigations in even more detail in the future.

Acknowledgements. HMI data are courtesy of NASA/SDO and the HMI science team. They are provided by the Joint Science Operations Center - Science Data Processing at Stanford University. The GOES X-ray flux measurements were made available by the National Geophysical Data Center. The SOHO/LASCO CME catalogue is maintained by the CDAW Data Center. The Vacuum Tower Telescope and ChroTel are operated by the Kiepenheuer-Institute for Solar Physics in Freiburg, Germany, at the Spanish Observatorio del Teide, Tenerife, Canary Islands. The ChroTel filtergraph has been developed by the KiepenheuerInstitute in cooperation with the High Altitude Observatory in Boulder, CO, USA. In particular, we would like to thank Dr. Thomas Kentischer, Clemens Halbgewachs, and Hans-Peter Doerr for their efforts to improve ChroTel and to ensure high-quality data. R.E.L. and C.D. were supported by grant DE 787/3-1 of the German Science Foundation (DFG). B.K. acknowledges the hospitality of the solar group at the Yunnan Astronomical Observatory, where most of his work was carried out, and the associated support by the Chinese Academy of Sciences under grant no. 2012T1J0017. He also acknowledges support by the DFG and the STFC. We are thankful to Drs. Christian Beck, Christian Bethge, and Christoph Kuckein for carefully reading the manuscript and providing valuable suggestions. We appreciate the inputs provided by Dr. Alexander Warmuth and Dr. Haimin Wang. We thank the anonymous referee and the editor for their helpful comments and very constructive suggestions.

\section{References}

Amari, T., Luciani, J. F., Aly, J. J., Mikic, Z., \& Linker, J. 2003, ApJ, 595, 1231 Amari, T., Aly, J.-J., Luciani, J.-F., Mikic, Z., \& Linker, J. 2011, ApJ, 742, L27 Anwar, B., Acton, L. W., Hudson, H. S., et al. 1993, Sol. Phys., 147, 287 Archontis, V., \& Hood, A. W. 2010, A\&A, 514, A56

Aschwanden, M. J. 2010, Sol. Phys., 262, 399

Aulanier, G., Török, T., Démoulin, P., \& DeLuca, E. E. 2010, ApJ, 708, 314

Bethge, C., Peter, H., Kentischer, T. J., et al. 2011, A\&A, 534, A105

Bethge, C., Beck, C., Peter, H., \& Lagg, A. 2012a, A\&A, 537, A130

Bethge, C., de Wijn, A. G., McIntosh, S. W., Tomczyk, S., \& Casini, R. 2012b, AAS Meeting Abstracts, 220, 13506

Brown, D. S., Nightingale, R. W., Alexander, D., et al. 2003, Sol. Phys., 216, 79 Burtseva, O., \& Petrie, G. 2013, Sol. Phys., 283, 429

Chen, P. F., \& Shibata, K. 2000, ApJ, 545, 524

Demoulin, P., van Driel-Gesztelyi, L., Schmieder, B., et al. 1993, A\&A, 271, 292

Deng, N., Xu, Y., Yang, G., et al. 2006, ApJ, 644, 1278

Denker, C. 2010, AN, 331, 648

Denker, C., \& Wang, H. 1998, ApJ, 502, 493

Emslie, A. G., Dennis, B. R., Shih, A. Y., et al. 2012, ApJ, 759, 71

Feynman, J., \& Martin, S. F. 1995, J. Geophys. Res., 100, 3355

Fisher, G. H., \& Welsch, B. T. 2008, in Subsurface and Atmospheric Influences on Solar Activity, eds. R. Howe, R. W. Komm, K. S. Balasubramaniam, \& G. J. D. Petrie, ASP Conf. Ser., 383, 373

Forbes, T. 2010, in Heliophysics: Space Storms and Radiation: Causes and Effects, eds. C. J. Schrijver, \& G. L. Siscoe (Cambridge, UK: Cambridge University Press), 159 
R. E. Louis et al.: Sunspot splitting triggering an eruptive flare

Forbes, T. G., \& Priest, E. R. 1995, ApJ, 446, 377

Garcia de La Rosa, J. I. 1987, Sol. Phys., 112, 49

Gary, G. A., \& Hagyard, M. J. 1990, Sol. Phys., 126, 21

Green, L. M., Kliem, B., \& Wallace, A. J. 2011, A\&A, 526, A2

Hagyard, M. J., Venkatakrishnan, P., \& Smith, Jr., J. B. 1990, ApJS, 73, 159

Hale, G. E., \& Nicholson, S. B. 1938, Magnetic observations of sunspots 1917 (Washington, D.C.: Carnegie Institution of Washington)

Handy, B. N., Acton, L. W., Kankelborg, C. C., et al. 1999, Sol. Phys., 187, 229

Harvey, K. L. 1985, Aust. J. Phys., 38, 875

Herdiwijaya, D., Makita, M., \& Anwar, B. 1997, PASJ, 49, 235

Inhester, B., Birn, J., \& Hesse, M. 1992, Sol. Phys., 138, 257

Jiang, Y., Zheng, R., Yang, J., et al. 2012, ApJ, 744, 50

Karpen, J. T., Antiochos, S. K., \& DeVore, C. R. 2012, ApJ, 760, 81

Kentischer, T. J., Bethge, C., Elmore, D. F., et al. 2008, in Advanced Software and Control for Astronomy II, eds. I. S. McLean, \& M. M. Casali, Proc. SPIE, 7014, 36

Kliem, B., \& Török, T. 2006, Phys. Rev. Lett., 96, 255002

Kliem, B., Su, Y., van Ballegooijen, A., \& DeLuca, E. 2013, ApJ, 779, 129

Kovacs, A., \& Dezso, L. 1986, Adv. Space Res., 6, 2

Kubo, M., Yokoyama, T., Katsukawa, Y., et al. 2007, PASJ, 59, 779

Kusano, K., Bamba, Y., Yamamoto, T. T., et al. 2012, ApJ, 760, 31

Leka, K. D., van Driel-Gesztelyi, L., Nitta, N., et al. 1994, Sol. Phys., 155, 301

Lemen, J. R., Title, A. M., Akin, D. J., et al. 2012, Sol. Phys., 275, 17

Li, H., Sakurai, T., Ichimoto, K., \& UeNo, S. 2000, PASJ, 52, 465

Lin, J., \& Forbes, T. G. 2000, J. Geophys. Res., 105, 2375

Lites, B. W. 2005, ApJ, 622, 1275

Liu, Y., \& Schuck, P. W. 2012, ApJ, 761, 105

Livi, S. H. B., Martin, S., Wang, H., \& Ai, G. 1989, Sol. Phys., 121, 197

Louis, R. E., Ravindra, B., Mathew, S. K., et al. 2012, ApJ, 755, 16

Low, B. C. 1996, Sol. Phys., 167, 217

Mackay, D. H., \& van Ballegooijen, A. A. 2006, ApJ, 641, 577

Mackay, D. H., Karpen, J. T., Ballester, J. L., Schmieder, B., \& Aulanier, G. 2010, Space Sci. Rev., 151, 333

MacTaggart, D., \& Hood, A. W. 2010, ApJ, 716, L219

Manchester, W., IV, Gombosi, T., DeZeeuw, D., \& Fan, Y. 2004, ApJ, 610, 588

Martin, S. F. 1998, Sol. Phys., 182, 107

Martin, S. F., Dezso, L., Antalova, A., Kucera, A., \& Harvey, K. L. 1982, Adv. Space Res., 2, 39

Martres, M.-J., Michard, R., Soru-Iscovici, I., \& Tsap, T. T. 1968, Sol. Phys., 5, 187

Mathew, S. K., \& Ambastha, A. 2000, Sol. Phys., 197, 75

Min, S., \& Chae, J. 2009, Sol. Phys., 258, 203

Murphy, G. A. 1990, Cooperative Thesis No. 124, University of Sydney and National Center for Atmospheric Research (NCAR/CT-124), Australia

Neupert, W. M. 2011, Sol. Phys., 272, 319
November, L. J. 1986, Appl. Opt., 25, 392

November, L. J., \& Simon, G. W. 1988, ApJ, 333, 427

Pesnell, W. D., Thompson, B. J., \& Chamberlin, P. C. 2012, Sol. Phys., 275, 3

Puschmann, K. G., Balthasar, H., Beck, C., et al. 2012a, in Ground-based and Airborne Instrumentation for Astronomy IV, Proc. SPIE, 8446, 79

Puschmann, K. G., Denker, C., Kneer, F., et al. 2012b, Astron. Nachr., 333, 880 Puschmann, K. G., Denker, C., Balthasar, H., et al. 2013, Opt. Eng., 52, 081606 Rempel, M. 2011a, ApJ, 729, 5

Rempel, M. 2011b, ApJ, 740, 15

Rempel, M., Schüssler, M., \& Knölker, M. 2009, ApJ, 691, 640

Rust, D. M. 1972, Sol. Phys., 25, 141

Sakajiri, T., Brooks, D. H., Yamamoto, T., et al. 2004, ApJ, 616, 578

Savcheva, A. S., Green, L. M., van Ballegooijen, A. A., \& DeLuca, E. E. 2012, ApJ, 759, 105

Schmieder, B., Hagyard, M. J., Guoxiang, A., et al. 1994, Sol. Phys., 150, 199

Schou, J., Scherrer, P. H., Bush, R. I., et al. 2012, Sol. Phys., 275, 229

Schrijver, C. J. 2009, Adv. Space Res., 43, 739

Schrijver, C. J., \& Title, A. M. 1999, Sol. Phys., 188, 331

Schrijver, C. J., De Rosa, M. L., Metcalf, T., et al. 2008, ApJ, 675, 1637

Schröter, E. H., Soltau, D., \& Wiehr, E. 1985, Vistas in Astron., 28, 519

Schüssler, M., \& Rempel, M. 2005, A\&A, 441, 337

Sterling, A. C., Chifor, C., Mason, H. E., Moore, R. L., \& Young, P. R. 2010, A\&A, 521, A49

Su, Y., Surges, V., van Ballegooijen, A., DeLuca, E., \& Golub, L. 2011, ApJ, 734,53

Sun, X., Hoeksema, J. T., Liu, Y., et al. 2012, ApJ, 748, 77

Švanda, M., Klvaňa, M., \& Sobotka, M. 2009, A\&A, 506, 875

Tan, C., Chen, P. F., Abramenko, V., \& Wang, H. 2009, ApJ, 690, 1820

Török, T., Temmer, M., Valori, G., et al. 2013, Sol. Phys., 286, 453

van Ballegooijen, A. A \& Martens, P. C. H. 1989, ApJ, 343, 971

van Driel-Gesztelyi, L., \& Petrovay, K. 1990, Sol. Phys., 126, 285

Vargas Domínguez, S., MacTaggart, D., Green, L., van Driel-Gesztelyi, L., \& Hood, A. W. 2012, Sol. Phys., 278, 33

Wang, H. 2006, ApJ, 649, 490

Wang, J., \& Shi, Z. 1993, Sol. Phys., 143, 119

Wang, H., Tang, F., Zirin, H., \& Ai, G. 1991, ApJ, 380, 282

Welsch, B. T., Fisher, G. H., Abbett, W. P., \& Regnier, S. 2004, ApJ, 610, 1148

Yan, X.-L., Qu, Z.-Q., Xu, C.-L., Xue, Z.-K., \& Kong, D.-F. 2009, Res. Astron. Astrophys., 9, 596

Yan, X. L., Qu, Z. Q., Kong, D. F., \& Xu, C. L. 2012, ApJ, 754, 16

Yang, J., Jiang, Y., Yang, B., et al. 2012, New Astron., 17, 732

Zhang, J., Wang, J., Deng, Y., \& Wu, D. 2001, ApJ, 548, L99

Zhang, J., Li, L., \& Song, Q. 2007, ApJ, 662, L35

Zhang, Y., Liu, J., \& Zhang, H. 2008a, Sol. Phys., 247, 39

Zhang, Y., Zhang, M., \& Zhang, H. 2008b, Sol. Phys., 250, 75 